\title{
Hydrodynamic theory for quantum plasmonics: Linear-response dynamics of the inhomogeneous electron gas
}

\author{
Yan, Wei
}

Published in:

Physical Review B

Link to article, DOI:

10.1103/PhysRevB.91.115416

Publication date:

2015

Document Version

Publisher's PDF, also known as Version of record

Link back to DTU Orbit

Citation (APA):

Yan, W. (2015). Hydrodynamic theory for quantum plasmonics: Linear-response dynamics of the inhomogeneous electron gas. Physical Review B, 91(11), [115416].

https://doi.org/10.1103/PhysRevB.91.115416

\section{General rights}

Copyright and moral rights for the publications made accessible in the public portal are retained by the authors and/or other copyright owners and it is a condition of accessing publications that users recognise and abide by the legal requirements associated with these rights.

- Users may download and print one copy of any publication from the public portal for the purpose of private study or research.

- You may not further distribute the material or use it for any profit-making activity or commercial gain

- You may freely distribute the URL identifying the publication in the public portal

If you believe that this document breaches copyright please contact us providing details, and we will remove access to the work immediately and investigate your claim. 


\title{
Hydrodynamic theory for quantum plasmonics: Linear-response dynamics of the inhomogeneous electron gas
}

\author{
Wei Yan* \\ DTU Fotonik, Department of Photonics Engineering, Technical University of Denmark, DK-2800 Kongens Lyngby, Denmark \\ and Center for Nanostructured Graphene, Technical University of Denmark, DK-2800 Kgs. Lyngby, Denmark \\ (Received 2 December 2014; revised manuscript received 6 February 2015; published 12 March 2015)
}

\begin{abstract}
We investigate the hydrodynamic theory of metals, offering systematic studies of the linear-response dynamics for an inhomogeneous electron gas. We include the quantum functional terms of the Thomas-Fermi kinetic energy, the von Weizsäcker kinetic energy, and the exchange-correlation Coulomb energies under the local density approximation. The advantages, limitations, and possible improvements of the hydrodynamic theory are transparently demonstrated. The roles of various parameters in the theory are identified. We anticipate that the hydrodynamic theory can be applied to investigate the linear response of complex metallic nanostructures, including quantum effects, by adjusting theory parameters appropriately.
\end{abstract}

DOI: 10.1103/PhysRevB.91.115416

PACS number(s): 78.20.Ci, 71.45.Gm, 42.70.Qs, 71.45.Lr

\section{INTRODUCTION}

Plasmon resonances, i.e., collective oscillations of free charge carriers, have the appealing property of being free of the diffraction limitation of optical waves, and confining the electric fields to the sub-(optical)wavelength scale with extremely high intensities [1-3]. Fundamental plasmon phenomena and the continuously developing nanotechnology are fueling further developments in the fields of nanoplamonics, metamaterials, and nanophotonics. The emerging novel applications include cancer therapy [4], surface enhanced raman spectroscopy to single molecule level $[5,6]$, nanolasers $[7,8]$, and quantum information processing [9].

Plasmonic materials cover a wide range including typical conducting media such as metals and doped semiconductors [10,11], and also low-dimensional materials such as graphene [12,13]. Different materials share the common physical picture of the free charge carrier gas mediated by Coulomb interactions in a polarizable or nonpolarizable background [10,11]. Guaranteed by the Kohn-Hohenberg theorem of the density-functional theory (DFT) $[14,15]$ and the Runge-Gross theorem of the time-dependent density functional theory (TDDFT) [16,17], the dynamics of the system can be described by two macroscopic quantities: the average particle density $n(\mathbf{r}, t)$ and the average particle current density $\mathbf{j}(\mathbf{r}, t)$ self-consistently with $[18,19]$

$$
\begin{aligned}
\frac{\partial n(\mathbf{r}, t)}{\partial t} & =-\nabla \cdot \mathbf{j}(\mathbf{r}, t), \\
m_{c} \frac{\partial \mathbf{j}(\mathbf{r}, t)}{\partial t} & =n(\mathbf{r}, t) q_{c} \mathbf{E}(\mathbf{r}, t)-\nabla \cdot \mathbf{P}(\mathbf{r}, t),
\end{aligned}
$$

where $m_{c}$ and $q_{c}$ represent the mass and charge of a single particle, $\mathbf{E}$ represents the electric field, and $\mathbf{P}$ - the key termis a tensor including all internal many-body quantum effects. The explicit form of $\mathbf{P}$ is not known, and different choices of $\mathbf{P}$ depending on the system size scale $l$ lead to different level theories. If $l \gg \lambda_{c}$, where $\lambda_{c}$ represents the characteristic wavelength of the carrier, one can safely neglect $\mathbf{P}$ and arrive

\footnotetext{
*wyan@fotonik.dtu.dk
}

at the local Drude theory (LDT), which earns the credits in the large-scale regime [1,2]. The ongoing progressions in nanofabrications push the size scale down to the deep nanometer with $l \sim \lambda_{c}$ [20-26]. This is the regime where the quantum wave nature of free electron gas, i.e., the term $\mathbf{P}$, starts to modify the plasmon response via two important effects, the nonlocality of the electric permittivity in the Thomas-Fermi screening scale and the electron spill-out in the work function scale. In this regime, people mainly rely on two approaches of dealing with P. One approach is DFT and TDDFT to describe $\mathbf{P}$ by (noninteracting) many-body Schrödinger equations (SEs) [27-29]. The other approach is the hydrodynamic theory (HT) [30-33] or quantum-continuous mechanics [18,19], where one approximates $\mathbf{P}$ by a simple functional expression.

The simplest version of the HT relies on the ThomasFermi (TF) theory, where $\mathbf{P}$ only includes the TF kinetic energy term [34-37]. The Thomas-Fermi hydrodynamic theory (TFHT) is usually combined with the assumptions of an infinitely large work function and a uniform equilibrium charge density. In other words, the electrons cannot escape the metal volume and in equilibrium they are distributed uniformly throughout the metal. Mathematically, the infinitely large work function leads to a hard-wall boundary condition (HWBC). Within this framework, the electron spill-out is neglected. This is in spirit close to the widely used local-response approximation [1].

Comparing with the DFT and TDDFT, the TFHT gains its advantage of numerical efficiency [38-40], however, suffers from the blame of the inaccuracy especially for the simple metals due to the neglect of the electron spill-out $[28,29]$. Combined with advanced numerical techniques, such as the finite-element method [38,39,41] or the Green function surface-integral method [42-45], the HT can be applied to relatively large and complex plasmonic structures that are beyond practical reach for the DFT and TDDFT. Additionally, for the symmetrical structures, such as cylinder and sphere, the analytical analysis can be possible [46-50]. In this way, the TFHT is able to get quantum insight of nonlocal effects in structures, that are on the one hand too small to obey classical electrodynamics while on the other hand still too large for a full quantum-mechanical treatment to be feasible. 
Recently, the TFHT has been employed to successfully explain experimentally observed phenomena, such as the blueshift of the surface plasmon (SP) resonance of silver nanoparticles for the dimension decreasing [25], and the hybridization of the SP resonance between gold nanoparticles and plane interface with gaps down to the Ångstrom scale [24]. However, when dealing with the monomers of simple metals such as sodium or aluminium, or dimers of metallic structures with a sufficiently narrow gap to potentially support quantumwave-function overlaps and tunneling, the HWBC neglects exactly electron spill-out effects [28,29], where a diffusive electron profile outside the metal is needed. It seems that the HT itself cannot get out of such disadvantage, and other semiclassical theories outside the scope of the hydrodynamic framework, such as the quantum corrected model for the dimer structure, are needed [51]. We emphasis that this is an issue entirely related to the HWBC, rather than the TFHT itself. Relaxing the HWBC and imposing a smooth equilibrium electron density, the electron spill-out can be characterized by the TFHT [52,53]. However, such a scheme suffers from the danger of supporting the unphysical plasmon modes, and an equilibrium electron density consistent with the HT dynamics is needed for suppressing the spurious force [54]. The TFHT can actually predict a smooth electron profile self-consistently. However, it leads to a vanishing work function [55], implying that a bounded electron can be excited to a propagating one at any frequency, which is of course unphysical. Fortunately, with little effort, the unphysical part can be overcome by including the first-order correction to the TF kinetic energy term due to the inhomogeneity of the equilibrium charge density. This correction is known as the von Weizsäcker (VW) term [56,57] and one may add the exchange and correlation Coulomb corrections for the further improvement of accuracy [54,58]. Such extended HT is usually referred to by the abbreviation TFVWHT [54] or alternatively the quantum-hydrodynamic theory (QHT) $[59,60]$. In this paper we will adapt the latter name. Of course, there are many further refinements of the HT that go beyond the QHT $[18,19,61-66]$. Obviously, the more advanced theory usually involves a more complicated $\mathbf{P}$, which makes the numerical investigation even more expensive. On the other hand, the community of nanoplasmonics is largely interested in the leading-order correction to the classical electrodynamics and with a call for efficient and faithful numerical tools. Our choice in this trade-off is the QHT.

In the present paper, we focus on the QHT for characterizing the linear-response dynamics (LRD) of an inhomogeneous electron gas in metallic media including both nonlocality and spill-out effects. The literature is rich on contributions to this field. It has been demonstrated that the QHT can predict the work function and surface energy well $[58,67]$, capture the important features of the linear excitations of free electron gas [54], and predict the nonlinear dynamics [68]. Recently, the QHT has been employed to investigate the surface plasmon response of two-dimensional (2D) nanowires of both simple metal (sodium) and noble metal (silver), and successfully predicts the dimension-dependent SP resonance shift [69]. However, a comprehensive understanding of the QHT is still needed. It is not clear what the specific limitation of the theory is, how one can adjust theory parameters for optimally characterizing the surface plasmon response especially in the high frequency, and how one could further improve the
QHT. In the present paper, we aim to clarify these somewhat unclear points, and wish to demonstrate both advantages and disadvantages of the theory transparently. For the convenience of the analysis, we mainly focus on the simple metals with the semi-infinite structure.

The remaining part of the paper is organized as follows. Section II introduces the QHT following the routine of the semiclassical Hamiltonian approach [36,37]. Section III analyzes the ground-state electron density and compares the results of the QHT and DFT. It is noted that the derived hydrodynamic equations in Sec. II and the numerical results in Sec. III are not completely new, but rather they serve to give a self-contained account of the theory. The insight in Sec. III is to offer a systematical comparison of the electron density in both the bulk and low electron density regions of the DFT and QHT analytically, and suggest the spatial dependence of the von Weizsäcker parameter. The LRD equation is introduced in Sec. IV. The LRD in a homogenous electron gas is discussed in Sec. V, and the frequency dependence of the von Weizsäcker (VW) parameter is demonstrated. In Sec. VI, we investigate the LRD in a weakly inhomogeneous electron gas, and suggest a new correction term and a slight modification to the theory in the high-frequency regime based on the microscopic considerations. In Sec. VII, the LRD in the electron-tail region is discussed. It is found that mathematically the QHT supports four longitudinal modes among which two modes are justified to be physical. Further, we interpret the two physical modes, and show their close relation with the photoelectric effect. In Sec. VII, the remarks on the findings of Secs. V-VII are given to better guide the readers. In Sec. IX, we define a both physically and mathematically correct boundary condition (BC) for the LRD. In Sec. X, the LRD is investigated in great detail by numerical analysis, and the results from the QHT are compared with the quantum mechanical calculations by the time-dependent local-density approximation (TDLDA) method. It is highlighted that a universal relation between the multipole surface-plasmon resonance and the threshold frequency of the photoelectric effect is shown and proven, and a useful quantity called opposite centroid frequency region is defined for identifying the qualitatively validity region of the QHT. Finally, Sec. XI summarizes the paper. Some detailed derivations and discussions are given in Appendixes A and B.

\section{HYDRODYNAMIC DESCRIPTION}

Macroscopic hydrodynamic theory for a free electron gas can be derived from different starting points, such as the semiclassical Hamiltonian including the quantum functional energy [36,37], the Boltzmann equation [70], the one-particle Winger distribution function $[59,60]$ or even quantum many-body SEs [18,19]. Here, from the semiclassical Hamiltonian [36,37], we outline the route to the QHT. The semiclassical Hamiltonian in the absence of a magnetic field is $[36,37]$

$$
\begin{aligned}
\mathcal{H}= & \frac{1}{2} m_{e} \int d^{3} \mathbf{r} n(\mathbf{r}, t)|\nabla S(\mathbf{r}, t)|^{2}+G[n(\mathbf{r}, t)] \\
& +\frac{1}{2} e^{2} \int d^{3} \mathbf{r} d^{3} \mathbf{r}^{\prime} \frac{n(\mathbf{r}, t) n\left(\mathbf{r}^{\prime}, t\right)}{4 \pi \epsilon_{0}\left|\mathbf{r}-\mathbf{r}^{\prime}\right|} \\
& -e \int d^{3} \mathbf{r} n(\mathbf{r}, t)\left[V_{\mathrm{ion}}(\mathbf{r})+V_{\mathrm{ext}}(\mathbf{r}, t)\right],
\end{aligned}
$$


where $S(\mathbf{r}, t)$ is the scalar velocity potential which relates to the velocity field $\mathbf{v}(\mathbf{r}, t)$ by $\mathbf{v}=-\nabla S$. Furthermore, $n(\mathbf{r}, t)$ is the density of the electron gas to be determined, $G[n(\mathbf{r}, t)]$ accounts for the internal many-body quantum energy, $V_{\text {ion }}(\mathbf{r})$ denotes the static electric potential from the positive ion background with a constant density $n_{\text {ion }}$ inside the metal, and $V_{\text {ext }}(\mathbf{r}, t)$ refers to the external electric potential. We note that the functional $G$ usually includes the following contributions: the internal kinetic energy, the exchange Coulomb energy, and the correlation Coulomb energy. Assuming that $n(\mathbf{r}, t)$ is canonically conjugated with $m_{e} S$, the Hamiltonian's equations (HEs) for the system are

$$
\begin{aligned}
& \nabla \cdot(n \mathbf{v})=-\partial n / \partial t, \\
& m_{e} \frac{d \mathbf{v}}{d t}=-\nabla \frac{\delta G}{\delta n}-e \nabla \phi,
\end{aligned}
$$

where $\phi$ represents the total electric potential felt by a single electron and has the expression,

$$
\phi=-e \int d^{3} r^{\prime} \frac{n\left(\mathbf{r}^{\prime}, t\right)}{4 \pi \epsilon_{0}\left|\mathbf{r}-\mathbf{r}^{\prime}\right|}+V_{\mathrm{ion}}(\mathbf{r})+V_{\mathrm{ext}}(\mathbf{r}, t) .
$$

The HEs can be employed to investigate our main interest, i.e., the LRD of the system responding to the external electric potential $V_{\text {ext }}$. To proceed, we assume a weak external perturbation. In the according linearization, the various fields are expressed as $n(\mathbf{r}, t)=n_{0}(\mathbf{r})+n_{1}(\mathbf{r}, t), \phi(\mathbf{r}, t)=\phi_{0}(\mathbf{r})+\phi_{1}(\mathbf{r}, t)$, $\mathbf{v}(\mathbf{r}, t)=\mathbf{v}_{1}(\mathbf{r}, t)$, and $\delta G / \delta n=(\delta G / \delta n)_{0}+(\delta G / \delta n)_{1}$. Here, the quantities with the subscript " 0 " refer to the unperturbed equilibrium case (the ground state), while the subscript " 1 " represents the small nonequilibrium corrections associated with the external driving. Clearly, this implies that the equilibrium electron density $n_{0}$ should satisfy

$$
\left(\frac{\delta G}{\delta n}\right)_{0}+e \phi_{0}=\mu
$$

where the chemical potential $\mu$ is constant throughout space. In this way, the linearized equations for the excited electron density $n_{1}$ and the electron charge current $\mathbf{J}_{1}=-e n_{0} \mathbf{v}_{1}$ become

$$
\begin{aligned}
\nabla \cdot \mathbf{J}_{1} & =e \frac{\partial n_{1}}{\partial t}, \\
\left(\frac{\partial}{\partial t}+\gamma\right) \mathbf{J}_{1} & =-\epsilon_{0} \omega_{p}^{2} \nabla \phi_{1}+\frac{n_{0} e}{m_{e}} \nabla\left(\frac{\delta G}{\delta n}\right)_{1},
\end{aligned}
$$

where $\omega_{p}=\sqrt{n_{\text {ion }} e^{2} / m_{e} \epsilon_{0}}$ represents the plasma frequency of the bulk metal, and the phenomenological damping rate $\gamma$ is added by hand. Equations (4a) and (4b) are the hydrodynamic equations determining the LRD of the system.

\section{A. Functional energy}

The quantum functional energy $G$ plays a central role in the QHT, and is expressed by the integral,

$$
G=\int d \mathbf{r} g[n(\mathbf{r}, t)]
$$

where $g$ is given by

$$
\begin{aligned}
g= & \frac{\hbar^{2} \pi^{3}}{10 m_{e}}\left(\frac{3 n}{\pi}\right)^{5 / 3}+\frac{\lambda_{w} \hbar^{2}}{8 m_{e}} \frac{\nabla n \cdot \nabla n}{n} \\
& -0.0588 \frac{e^{2} n^{4 / 3}}{\epsilon_{0}}-\frac{0.035}{0.6024+7.8 a_{H} n^{1 / 3}} \frac{e^{2} n^{4 / 3}}{\epsilon_{0}},
\end{aligned}
$$

with $a_{H}=0.529 \AA$ being the Bohr radius. The first term of $g$ is known as the Thomas-Fermi (TF) term [71,72], which represents the quantum-kinetic energy for a uniform electron gas. The second term is the von Weizsäcker (VW) term or the quantum-pressure energy term [56,60], which gives the leading-order correction to the TF term due to the inhomogeneity. The third and fourth terms represent Wigner's exchange and correlation energies, respectively, under the local-density approximation (LDA) [73].

For the quantum functional energy, the TF and VW terms are the most important ones. In essence, the HT and the more advanced DFT and TDDFT differ by those terms. In particular, in the DFT or TDDFT, the quantum-kinetic energy is more precisely included in the (noninteracting) many-body SEs.

The TF term is valid under the local-equilibrium approximation (LEA) [74] that the electron gas obeys Fermi-Dirac statistics locally. The LEA holds in the low-frequency regime where $\omega \ll \gamma$, since the damping can relax the perpetuated electrons to the local-equilibrium configuration quickly. However, in the high-frequency regime where $\omega \gg \gamma$, such approximation breaks down. In this sense, a HT beyond the local equilibrium is needed [63,64]. In the bulk region of the metal, where the electron gas is nearly homogenous, this could be achieved by a slight modification to the theory [70]. However, in the surface region (the region near the metal boundary), where the electron gas varies on the atomic scale and interesting dynamics occur, it is challenging to extend the QHT beyond the LEA. In the present paper, for simplicity, we will restrict our discussion to the LEA. As demonstrated later, despite the problematic assumption of the LEA in the high-frequency regime, it still allows qualitatively correct predictions.

For the VW term, the parameter $\lambda_{w}$ is critical. For a weakly inhomogeneous electron gas, it is well known that $\lambda_{w}=1 / 9$ in the low-frequency regime [14,37], while it approaches 1 as the frequency increasing (demonstrated in Sec. V B). Additionally, for a single quantum state, we have $\lambda_{w}=1[30,60]$. This somehow suggests that $\lambda_{w}$ should approach 1 in the electrontail region (the region far outside the metal). The reason is that the response in the electron-tail region is dominated by quantum state near the Fermi level, which resembles the single state. The same conclusion can also be reached from different perspectives in Secs. III and VII. In summary, $\lambda_{w}$ should depend on frequency and vary in space. However, the exact dependence is not clear in the surface region (the region around the metal boundary). To work around this obstacle, one usually simply sets $\lambda_{w}$ to be a constant between 1 and $1 / 9$ [54,58-60,67,68]. This is also the chosen scheme in the present paper.

The hydrodynamic theory with $g$ only containing the TF term represents the simplest hydrodynamic theory, i.e., TFHT. Here, we note that, in the QHT, there are three basic parameters 
namely $n_{\text {ion }}, \gamma$ (damping for the LRD), and $\lambda_{w}$, which must be known before specific analysis and numerical computations. For the TFHT, the parameter $\lambda_{w}$ is not required due to the absence of the VW term, and accordingly there are two basic parameters. The main differences between QHT and TFHT will be discussed.

\section{GROUND-STATE ELECTRON DENSITY}

To predict the LRDs by the QHT, the equilibrium electron density $n_{0}$ is needed. In this section, we illustrate the numerical computation of $n_{0}$. Besides that, the analytical comparisons of the electron density in the bulk and electron-tail regions by the DFT and QHT are given, and the spatial dependence of $\lambda_{w}$ is suggested.

\section{A. Equations}

First, we define the following quantities: $E_{F}$ the Fermi energy of the uniform electron gas with the density equal to $n_{\text {ion }} ; v_{F}$ the Fermi velocity which relates to the Fermi energy through a parabolic dispersion relation $E_{F}=m_{e} v_{F}^{2} / 2$. We relate $n_{0}, \phi_{0}$, and $\mu$ in Eq. (3) with the following dimensionless quantities $f_{0}, \phi_{0}^{\prime}$, and $\mu^{\prime}$ by $f_{0}=\sqrt{n_{0} / n_{\text {ion }}}$, $\phi_{0}^{\prime}=\phi_{0} \epsilon_{0} k_{\mathrm{TF}}^{2} / e n_{\text {ion }}$ where $k_{\mathrm{TF}}$ is defined below Eq. (6), and $\mu^{\prime}=\mu / E_{F}$. Then, Eq. (3) is expressed in a dimensionless form,

$$
f_{0}^{4 / 3}+C_{0} f_{0}^{4 / 3}-C_{1} f_{0}^{2 / 3}-C_{2} \frac{\nabla^{2} f_{0}}{f_{0}}-2 \phi_{0}^{\prime}=\mu^{\prime},
$$

where

$$
\begin{aligned}
& C_{0}=\frac{2}{3} \frac{0.035 \mathrm{X}_{1}}{\left(0.6024+\mathrm{X}_{1} f_{0}^{2 / 3}\right)^{2}} \frac{k_{\mathrm{TF}}^{2}}{n_{\mathrm{ion}}^{2 / 3}}, \\
& C_{1}=\frac{8}{3}\left(0.0588+\frac{0.035}{0.6024+\mathrm{X}_{1} f_{0}^{2 / 3}}\right) \frac{k_{\mathrm{TF}}^{2}}{n_{\mathrm{ion}}^{2 / 3}}, \\
& C_{2}=\frac{\lambda_{w}}{k_{F}^{2}}, \\
& k_{\mathrm{TF}}=\frac{\omega_{p}}{v_{F}}, \quad k_{\mathrm{F}}=\frac{m_{e} v_{F}}{\hbar},
\end{aligned}
$$

$\mathrm{X}_{1}=7.8 a_{H} n_{\text {ion }}^{1 / 3}$. For the TFHT, we simply set $C_{0}=C_{1}=$ $C_{2}=0$ in Eq. (6). Besides Eq. (6), $\phi_{0}^{\prime}$ and $f_{0}$ also relate to each other by Poisson's equation with

$$
\frac{1}{k_{\mathrm{TF}}^{2}} \nabla^{2} \phi_{0}^{\prime}=f_{0}^{2}-f_{\mathrm{ion}}^{2},
$$

where $f_{\text {ion }}=1$ in the metal region and $f_{\text {ion }}=0$ outside the metal. For a neutral system, the condition that the total charge of the electrons equals that of the positive ions should be specified.

\section{B. Numerical analysis}

Equations (6) and (7) constitute two coupled nonlinear equations. The equations can be solved by the finite-element method (FEM) utilizing the commercially available code COMSOL MULTIPHYSICS 4.3B efficiently. For this, one needs to transform Eqs. (6) and (7) into the corresponding weak
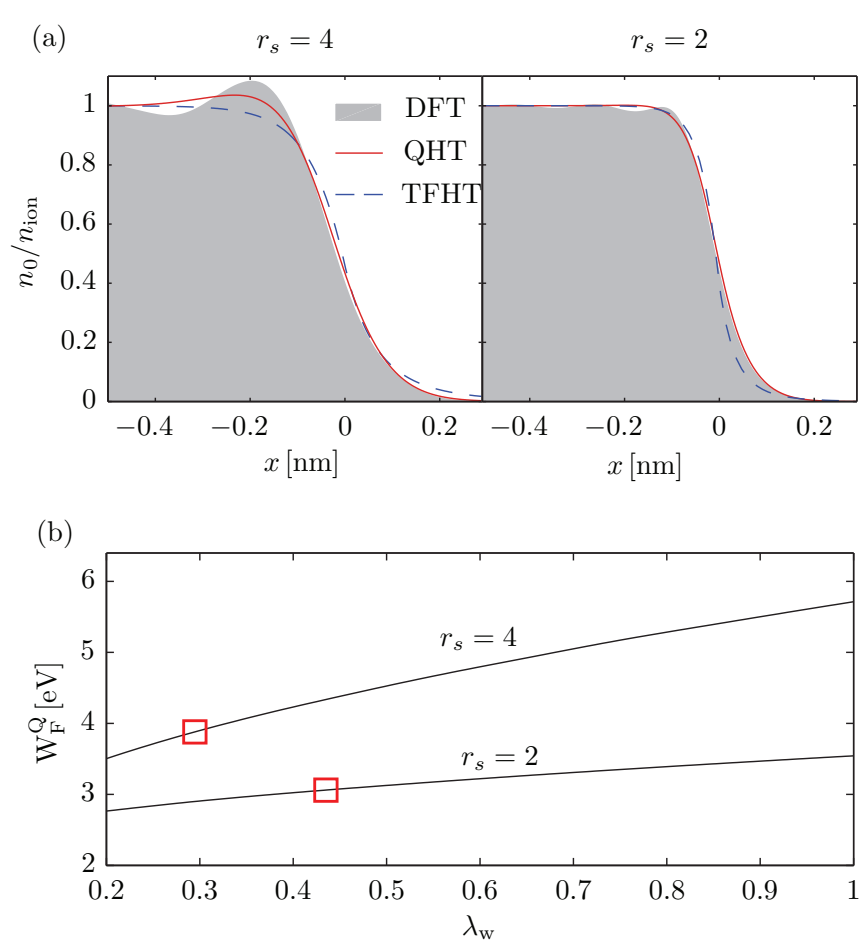

FIG. 1. (Color online) (a) The ground-state electron density $n_{0}$ for the simple metals with $r_{s}=4$ and $r_{s}=2$. The results are calculated by the TFHT, QHT, and DFT for the semi-infinite metal. In the QHT, we choose $\lambda_{w}=0.5$ for $r_{s}=4$, and $\lambda_{w}=0.25$ for $r_{s}=2$. (b) Work function versus $\lambda_{w}$ predicted by the QHT. The square markers represent the values of $\lambda_{w}$ giving the same work function as the DFT.

forms [75], then put them down in the weak-form environment of COMSOL MULTIPHYSICS, and solve them by the nonlinear iterative solver. The numerical complexity and speed of the QHT have obvious advantages over the DFT since the computation of the single particle eigenwave function is not needed. For the numerical illustration, we focus on the case that the metal is located in the semi-infinite space $x<0$. The scheme can also be applied to more complex geometries, such as cylinders demonstrated in Ref. [69].

Even though similar results can be found in Ref. [58], they are still given for demonstrating the validity of our FEM computation and also better understanding of the analytical analysis in Sec. IIIC. Two different metals with $r_{s}=4$ (sodium) and $r_{s}=2$ (aluminum) are considered. Here, the dimensionless lattice parameter $r_{s}$ is determined by $n_{\text {ion }}$ with $r_{s}=\left(3 / 4 \pi n_{\text {ion }}\right)^{1 / 3} / a_{H}$. For the TFHT and QHT calculations, we employ the FEM. The VW parameter $\lambda_{w}$ in the QHT is chosen to be $\lambda_{w}=0.5$ for $r_{s}=4$, and $\lambda_{w}=0.25$ for $r_{s}=2$. The particular values of $\lambda_{w}$ reproduce the electron density of the DFT reasonably. For the DFT calculations, we follow the numerical routine suggested by Kohn and Lang [55]. The results are plotted in Fig. 1(a). In the surface region around $x=0$, it is seen that the QHT has a better agreement with the DFT than the TFHT. In the bulk region, the density oscillations in the DFT are noticeable especially for the case $r_{s}=4$. However, for the QHT, the oscillations are nearly invisible due to the exponential damping behavior as analyzed in 
TABLE I. Approximate solutions of $f_{0}$ in the bulk region and the electron-tail region for the TFHT, QHT, and DFT. $a_{T, Q}, b_{T, Q, D}$, and $\eta_{Q, F}$ are constants.

\begin{tabular}{lcc}
\hline \hline Models & $f_{0 b}$ (Bulk region) & $f_{0 t}$ (Electron-tail region) \\
\hline TFHT & $1+a_{T} e^{\sqrt{1 / 3} k_{\mathrm{TF}} x}$ & $10^{1.5}\left(1+b_{T} / x\right) / x^{3}$ \\
QHT & $1+a_{Q} \cos \left(k_{\mathrm{LR}} x+\eta_{Q}\right) e^{k_{\mathrm{LI}} x}$ & $b_{Q} e^{-k_{Q} x}$ \\
DFT & $1+3 \cos \left(2 k_{F} x+\eta_{F}\right) / 8 k_{F}^{2} x^{2}$ & $b_{D} e^{-k_{D} x} / x$ \\
\hline \hline
\end{tabular}

Sec. III C, and only a small bump near $x=0$ can be observed. Additionally, we also calculate the work function. It is found that both the DFT and QHT predict a positive nonzero value, while the TFHT gives a zero value, agreeing with the analysis in Sec. IIIC. In Fig. 1(b), we plot the work function $W_{F}^{Q}$ predicted by the QHT as a function of $\lambda_{w}$. It is found that $\lambda_{w}$ around 0.435 for $r_{s}=4$, and $\lambda_{w}$ around 0.295 for $r_{s}=2$ give the work function close to the DFT value, as indicated by the square markers in the figure. The positions of the markers are close to the values of $\lambda_{w}$ chosen in Fig. 1(a), where decent agreements between the QHT and DFT have been observed.

\section{Analytical analysis}

It is hard to find analytical solutions of Eqs. (6) and (7) for complex geometries. For simplicity and without losing any important physics, we consider the semi-infinite metal structure as in Sec. III B. In this case, it becomes possible to find approximate analytic solutions.

In the bulk region, we denote $f_{0}$ by $f_{0 b}$, and express $f_{0 b}=1+f_{0 b}^{(1)}$, where $f_{0 b}^{(1)}$ accounts for the small spatially inhomogeneous correction. Treating $f_{0 b}^{(1)}$ as the perturbation term, and analyzing Eqs. (6) and (7), it is found that $f_{0 b}^{(1)}$ to the leading order satisfies the same equation as that for the linearly excited static longitudinal mode in the homogenous background, which will be discussed in Sec. V in detail. The wave number $k_{L}$ of the static longitudinal mode is determined by

$$
\epsilon_{m}^{L}\left(0, k_{L}\right)=0
$$

where $\epsilon_{m}^{L}$ represents the longitudinal permittivity, and is expressed in Eq. (11b). In the QHT, there are formally four complex-valued solutions for $k_{L}$. In the TFHT, there are formally only two solutions for $k_{L}$ given by the entirely imaginary expression $\pm i \sqrt{1 / 3} k_{\mathrm{TF}}$. With the boundary condition that $f_{0 b}^{(1)} \rightarrow 0$ as $x \rightarrow-\infty$, the general solution of $f_{0 b}^{(1)}$ can be written down. In Table I, we summarize the results for the TFHT, QHT, and also DFT. The DFT result is derived in Ref. [55]. It is seen that the QHT predicts the oscillations of the electron density in the bulk region like the DFT, but the oscillations decay much faster (exponentially decaying) than the DFT ( $1 / x^{2}$ decaying). In the TFHT, the oscillations disappear, which is attributed to the negligence of the VW term.

In the electron-tail region, we denote $f_{0}$ by $f_{0 t}$. In this region, $f_{0 t} \ll 1$. Accordingly, the first three terms on the right-hand side (RHS) of Eq. (6) are negligible, and Eq. (6) is approximate to

$$
\nabla^{2} f_{0 t}-\frac{W_{F}^{Q}}{C_{2} E_{F}} f_{0 t}=0,
$$

where $W_{F}^{Q}=-\lim _{x \rightarrow+\infty}\left(\mu+2 \phi_{0}\right)$ is the work function predicted by the QHT. The work function is the minimum energy needed to eject an electron from the bulk metal into the vacuum. With the boundary condition that $f_{0 t} \rightarrow 0$ as $x \rightarrow$ $+\infty$, we have $f_{0 t} \simeq b_{Q} \mathrm{e}^{-k_{Q} x}$, where $k_{Q}$ relates with $W_{F}^{Q}$ by

$$
W_{F}^{Q}=\lambda_{w} \frac{\hbar k_{Q}^{2}}{2 m_{e}},
$$

where $b_{Q}$ is a constant. However, for the TFHT, Eq. (6) directly implies that $\lim _{x \rightarrow+\infty}\left(\mu+2 \phi_{0}\right)=0$, i.e., a zero work function. Additionally, by the analysis to Eqs. (6) and (7) [76], the approximate solution of $f_{0 t}$ for the TFHT is found to be $f_{0 t} \simeq 10^{1.5}\left(1+b_{T} / x\right) / x^{3}$, where $b_{T}$ is a constant. For the DFT, the electron density can be expressed by the integration of the Kohn-Sham orbitals [55], i.e.,

$$
n_{0}(x)=\frac{1}{\pi} \int_{0}^{k_{F}} d k\left(k_{F}^{2}-k^{2}\right)\left|\psi_{k}(x)\right|^{2} .
$$

In the electron-tail region, $\psi_{k}$ is simply an exponentially decaying function, and the integration has the approximate solution $f_{0 t} \simeq b_{D} e^{-k_{D} x} / x$, where $b_{D}$ is a constant, and $k_{D}$ relates with the DFT work function $W_{F}^{D}$ by

$$
W_{F}^{D}=\frac{\hbar k_{D}^{2}}{2 m_{e}},
$$

which is of exactly the same form as Eq. (8), provided that $\lambda_{w}=1$. This indicates that $\lambda_{w}$ approaching 1 in the electron-tail region can result in a better agreement between the QHT and DFT. Together with the fact that $\lambda_{w}=1 / 9$ in the bulk region for the ground state [14,37], we expect that an improved QHT can be consistent with such spatial dependence of $\lambda_{w}$.

The approximate solutions of $f_{0 t}$ for the TFHT, QHT, and DFT are summarized in Table I. It is seen that the QHT and DFT share the same feature that $f_{0 t}$ contains the exponentially decaying term, except that an additional $1 / x$ term appears in the DFT. In the TFHT, $f_{0 t}$ is dominated by the $1 / x^{3}$ term, which decays much slower. In conclusion, the above results indicate that the QHT mimics the DFT better than the TFHT.

\section{EQUATIONS FOR LINEAR-RESPONSE DYNAMICS}

The LRD due to the external driving is determined by Eqs. (4a) and (4b). In particular, Eq. (4b) can be expressed in a more transparent form as

$$
\left(\frac{\partial}{\partial t}+\gamma\right) \frac{\partial \mathbf{J}_{1}}{\partial t}=\epsilon_{0} \omega_{p}^{2} f_{0}^{2} \frac{\partial \mathbf{E}_{1}}{\partial t}+\beta^{2}\left(\nabla-\frac{2 \nabla f_{0}}{f_{0}}\right) Q_{1},
$$

where

$$
Q_{1}=\left(N^{(0)}+\mathbf{N}^{(1)} \cdot \nabla-N^{(2)} \nabla^{2}\right) \nabla \cdot \mathbf{J}_{1},
$$


with

$$
\begin{aligned}
\beta= & \sqrt{\frac{1}{3}} v_{F}, \\
N^{(0)}= & f_{0}^{4 / 3}+C_{0} \frac{v_{F}^{2}}{\beta^{2}} f_{0}^{4 / 3}-\frac{C_{1}}{6} \frac{v_{F}^{2}}{\beta^{2}} f_{0}^{2 / 3}-\frac{C_{3}}{3} \frac{v_{F}^{2}}{\beta^{2}} f_{0}^{2} \\
& +2 N^{(2)}\left(\frac{\nabla^{2} f_{0}}{f_{0}}-\frac{\left|\nabla f_{0}\right|^{2}}{f_{0}^{2}}\right), \\
\mathbf{N}^{(1)}= & 2 N^{(2)} \frac{\nabla f_{0}}{f_{0}}, \\
N^{(2)}= & \frac{C_{2}}{4} \frac{v_{F}^{2}}{\beta^{2}}, \\
C_{3}= & \frac{2}{3} \frac{0.035 X_{1}^{2}}{\left(0.6024+X_{1} f^{2 / 3}\right)^{3}} \frac{k_{\mathrm{TF}}^{2}}{n_{\text {ion }}^{2 / 3}},
\end{aligned}
$$

and $\mathbf{E}_{1}$ represents the electric field. In Eq. (9), we have $\beta=$ $\sqrt{1 / 3} v_{F}$ as a result of the LEA. However, when $\omega \gg \gamma$, the LEA breaks down, and $\beta$ is accordingly modified to $\sqrt{3 / 5} v_{F}$ at least in the bulk region [70], which is discussed in Sec. V.

\section{LINEAR-RESPONSE DYNAMICS IN A HOMOGENOUS ELECTRON BACKGROUND}

Incorporating Eq. (9) with Maxwell's equations, the LRDs of the system can be predicted. Before going to the detailed numerics, we would like to investigate the rich physical indications of Eq. (9) by analytical analysis in Secs. V-VII for three different cases: a homogenous electron gas, a weakly inhomogeneous electron gas, and the electron-tail region. In this section, we focus on the homogenous case.

\section{A. Transverse and longitudinal permittivities}

Deep into the bulk region of the metal, the electron gas is nearly homogenous with $\nabla f_{0} \rightarrow 0$. In this case, decomposing the fields into the transverse and longitudinal components, and relating the electric field with the polarization field $\mathbf{P}_{1}\left(\mathbf{J}_{1}=\right.$ $\partial \mathbf{P}_{1} / \partial t$ ), we extract the familiar transverse and longitudinal permittivities [32],

$$
\begin{aligned}
\epsilon_{m}^{T}(\omega) & =1-\frac{\omega_{p}^{2}}{\omega^{2}+\mathrm{i} \omega \gamma}, \\
\epsilon_{m}^{L}\left(\omega, k_{L}\right) & =1-\frac{\omega_{p}^{2}}{\omega^{2}+\mathrm{i} \omega \gamma-\beta^{2} k_{L}^{2}\left(N^{(0)}+N^{(2)} k_{L}^{2}\right)} .
\end{aligned}
$$

The longitudinal modes are determined by $\epsilon_{m}^{L}\left(\omega, k_{L}\right)=0$. Neglecting the damping, we summarize the solutions of $k_{L}$ in Table II. For the QHT, the longitudinal modes support four solutions. When $\omega<\omega_{q}$ where

$$
\omega_{q}=\operatorname{Re}\left(\frac{\omega_{p}^{2}-N_{0}^{2} \beta^{2}}{4 N_{2}}\right),
$$

$k_{L}$ is complex valued, implying that the excited charges undergo damped oscillations rather than purely exponential decay. In the static case, such complex valued longitudinal modes determine the leading-order inhomogeneity of the ground-state electron density as discussed in Sec. III. When
TABLE II. Longitudinal wave numbers $k_{L}$ for the TFHT and QHT.

\begin{tabular}{lcc}
\hline \hline & \multicolumn{2}{c}{$k_{L}$} \\
\cline { 2 - 3 } Frequency & QHT & TFHT \\
$0<\omega<\omega_{q}$ & $\pm k_{\mathrm{LR}} \pm \mathrm{i} k_{\mathrm{LI}}$ & (2 solutions) \\
$\omega_{q}<\omega<\omega_{p}$ & $\pm \mathrm{i} k_{\mathrm{LI} 1} ; \pm \mathrm{i} k_{\mathrm{LI} 2}$ & $\pm \mathrm{i} k_{\mathrm{LI}}$ \\
$\omega>\omega_{p}$ & $\pm \mathrm{i} k_{\mathrm{LI}} ; \pm k_{\mathrm{LR}}$ & $\pm k_{\mathrm{LR}}$ \\
\hline \hline
\end{tabular}

$\omega_{q}<\omega<\omega_{p}, k_{L}$ turns entirely imaginary. When $\omega>\omega_{p}$, two of the four $k_{L}$ solutions become real values corresponding to longitudinal plasmon modes known to propagate in the bulk region of the metal. For the TFHT, we have $N^{(0)}=1$ and $N^{(2)}=0$ in Eq. (11b). In this case, only two solutions exist. Below the plasma frequency, $k_{L}$ solutions are entirely imaginary; above the plasma frequency, $k_{L}$ are completely real valued corresponding to propagating longitudinal modes.

In Fig. 2, we plot $k_{L}$ as a function of $\omega$ for the simple metal with $r_{s}=4$. In this figure, we choose $\beta=\sqrt{3 / 5} v_{F}$ and $\lambda_{w}=1$ according to the discussions in the following subsection. The observations in Fig. 2 are consistent with Table II.

\section{B. Discussions on $\beta$ and $\lambda_{w}$}

The parameters $\beta$ and $\lambda_{w}$ are of central importance in the QHT. Here, we want to see what specific values of $\beta$ and $\lambda_{w}$ can give the most accurate prediction of the LRD for the homogenous electron gas. For this, we compare the longitudinal permittivity in Eq. (11b) with that derived from the more advanced many-body random-phase approximation (RPA) method. In Ref. [70], the comparisons were performed to the second order of $k_{L}$, and the frequency-dependent expression of $\beta$ was given as a correction of the HT, which is especially important in the high frequency. Here, we extend the comparisons up to the fourth order of $k_{L}$, and derive the frequency-dependent expression of $\lambda_{w}$ as the main contribution in this subsection. Since the exchange and correlation interactions are neglected in the RPA formula, we here also neglect exchange and correlation interactions for the QHT by setting $N^{(0)}=1$ in Eq. (11b). The neglec is not important to $\beta$ and $\lambda_{w}$, because such two parameters originate

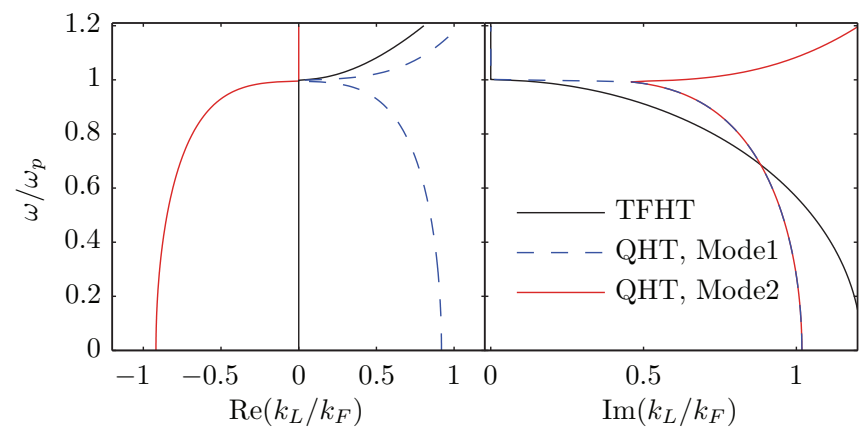

FIG. 2. (Color online) Longitudinal wave number $k_{L}$ versus frequency $\omega$ for the simple metal with $r_{s}=4$. Only the modes with $\operatorname{Im}\left(k_{L}\right) \geqslant 0$ are plotted. 
from the quantum kinetic energy (the TF and VW terms), not from the exchange and correlation interactions.

First, we consider the low-frequency regime where $\omega \ll \gamma$. If $\gamma=0$, this just reduces to the static case $\omega=0$. The static RPA permittivity for $k \ll k_{F}$ has the form [11],

$$
\epsilon_{m}^{R}\left(0, k_{L}\right) \simeq 1+\frac{3 k_{\mathrm{TF}}^{2}}{k_{L}^{2}}\left(1-\frac{1}{12} \frac{k_{L}^{2}}{k_{F}^{2}}\right),
$$

which agrees with Eq. (11b) to the same expansion order as long as $\beta=v_{F} \sqrt{1 / 3}$ and $\lambda_{w}=1 / 9$. Next, consider the highfrequency regime where $\omega \gg \gamma$, which is usually satisfied for $\omega$ at the optical frequency. The RPA [11] and QHT permittivities for $k_{L} \ll \omega / v_{F}$ have the following approximate expressions:

$$
\begin{aligned}
& \epsilon_{m}^{R}\left(\omega, k_{L}\right) \simeq 1-\frac{\omega_{p}^{2}}{\omega^{2}}\left[1+\frac{3 k_{L}^{2} v_{F}^{2}}{5 \omega^{2}}+\left(\frac{3}{7}+\frac{\omega^{2}}{4 v_{F}^{2} k_{F}^{2}}\right) \frac{k_{L}^{4} v_{F}^{4}}{\omega^{4}}\right], \\
& \epsilon_{m}^{L}\left(\omega, k_{L}\right) \simeq 1-\frac{\omega_{p}^{2}}{\omega^{2}}\left[1+\frac{k_{L}^{2} \beta^{2}}{\omega^{2}}+\left(\frac{\beta^{4}}{v_{F}^{4}}+\frac{\lambda_{w} \omega^{2}}{4 v_{F}^{2} k_{F}^{2}}\right) \frac{k_{L}^{4} v_{F}^{4}}{\omega^{4}}\right] .
\end{aligned}
$$

Clearly, if we set $\beta=\sqrt{3 / 5} v_{F}$ and $\lambda_{w}=1$, the above two expressions achieve a good agreement except the slight discrepancy occurs between the terms $3 / 7$ and $\beta^{4} / v_{F}^{4}=9 / 25$.

The above discussions indicate that both $\beta$ and $\lambda_{w}$ depend on frequency. In particular, we have $\beta=v_{F} \sqrt{1 / 3}$ and $\lambda_{w}=$ $1 / 9$ for $\omega \ll \gamma$, while $\beta=v_{F} \sqrt{3 / 5}$ and $\lambda_{w}=1$ for $\omega \gg \gamma$.

In the intermediate frequency region where $\omega$ is comparable with $\gamma, \beta$ and $\lambda_{w}$ are expected to depend on both $\omega$ and $\gamma$. In Ref. [70], Halevi derived such an expression for $\beta$ with

$$
\beta=\sqrt{\frac{3 \omega / 5+i \gamma / 3}{\omega+i \gamma}} .
$$

The equation gives the correct low- and high-frequency limits, i.e., $\beta=\sqrt{1 / 3} v_{F}$ for $\omega \ll \gamma$ and $\beta=\sqrt{3 / 5} v_{F}$ for $\omega \gg \gamma$. Here, following Halevi's recipe, we derive the expression for $\lambda_{w}$. Including the damping within Mermin's relaxation-time approximation scheme [74], the correct RPA permittivity formula becomes

$$
\epsilon_{m}^{R^{\prime}}\left(\omega, k_{L}\right)=1+\frac{\omega^{\prime}\left[\epsilon_{m}^{R}\left(\omega^{\prime}, k_{L}\right)-1\right]}{\omega+i \gamma\left[\epsilon_{m}^{R}\left(\omega^{\prime}, k_{L}\right)-1\right] /\left[\epsilon_{m}^{R}\left(0, k_{L}\right)-1\right]},
$$

where $\omega^{\prime}=\omega+i \gamma$, and the expression of $\epsilon_{m}^{R}$ can be found in the above. Employing Taylor's expansion to $\epsilon_{m}^{R^{\prime}}$ up to the fourth order of $k_{L}$, and matching the derived one with the QHT permittivity, $\lambda_{w}$ is founded to be

$$
\lambda_{w}=\frac{\omega+i \gamma / 9}{\omega+\mathrm{i} \gamma} .
$$

Equation (12) leads to $\lambda_{w}=1 / 9$ for $\omega \ll \gamma$, and $\lambda_{w}=1$ for $\omega \gg \gamma$.

\section{LINEAR-RESPONSE DYNAMICS IN A WEAKLY INHOMOGENEOUS BACKGROUND}

In Sec. V, we have shown that the QHT can describe the LRD in the homogenous electron gas well, and the two important parameters $\beta$ and $\lambda_{w}$ both depend on frequency. Next, it is natural to ask whether the same conclusion holds when the electron gas becomes inhomogeneous? Do the coefficients related to the inhomogeneity of the ground-state density in Eq. (9) have the correct forms? Obviously, these are in general difficult questions while insights into the weakly inhomogeneous electron gas may shed some light for constructing a better HT.

For simplicity, we assume that the electron gas is inhomogeneous only in the $x$ direction with $n_{0}(x)=n_{\text {ion }}+n_{\text {ih }}(x)$. Here, $n_{\text {ih }}$ represents the small inhomogeneity around $n_{\text {ion }}$ with $n_{\text {ih }} \ll n_{\text {ion }}$ and $\left|d n_{\text {ih }} / d x\right| / n_{\text {ih }} \ll k_{F}$. Furthermore, we assume that $n_{\text {ih }}$ is induced by some self-consistent static potential. Our strategy is to extract a linear-response equation from the microscopic considerations, and compare the derived equation with the QHT one. Employing the self-consistent field (SCF) method [10,77], the dynamics of the system is governed by the single-particle Liouville equation,

$$
[\hat{\mathcal{H}}, \hat{\rho}]=i \hbar \frac{\partial \hat{\rho}}{\partial t},
$$

where $\hat{\rho}$ is the operator associated with the single-particle density matrix, and $\hat{\mathcal{H}}$ represents the single-particle Hamiltonian expressed by

$$
\hat{\mathcal{H}}=\hat{\mathcal{H}}_{0}+V_{\mathrm{sc}}(x, t),
$$

with $\hat{\mathcal{H}}_{0}=-\hbar^{2} \nabla^{2} / 2 m_{e}$ representing the free-electron Hamiltonian and $V_{\mathrm{sc}}$ being the self-consistent potential. Further, we assume that $V_{\mathrm{sc}}$ only includes the electric potential, which simply corresponds to setting $C_{0}=C_{1}=C_{3}=0$ in Eq. (9). Next, we split $V_{\mathrm{sc}}$ into $V_{\mathrm{sc}}=V_{\mathrm{sc}, 0}+V_{\mathrm{sc}, \omega}$ with

$$
\begin{aligned}
& V_{\mathrm{sc}, 0}=\int d k v_{\mathrm{sc}, 0}(k) e^{\mathrm{i} k x}, \\
& V_{\mathrm{sc}, \omega}=\int d k v_{\mathrm{sc}, \omega}(k) e^{\mathrm{i} k x-\mathrm{i} \omega t},
\end{aligned}
$$

where $V_{\mathrm{sc}, 0}$ is the static potential which induces $n_{\mathrm{ih}}$, while $V_{\mathrm{sc}, \omega}$ represents the dynamic potential which excites the oscillating density $n_{\omega}$. Below, we will suppress the time dependence $e^{-\mathrm{i} \omega t}$ for ease of notation.

Treating $V_{\text {sc }}$ as the small perturbation, we approximate $\hat{\rho}$ as

$$
\hat{\rho} \simeq \hat{\rho}_{0}+\hat{\rho}_{0}^{(1)}+\left(\hat{\rho}_{\omega}^{(1)}+\hat{\rho}_{\omega}^{(2)}\right),
$$

where $\hat{\rho}_{0}$ represents the equilibrium single-particle density matrix, the superscripts "(1)" and "(2)" denote the first- and second-order correction terms due to $V_{\mathrm{sc}}$, respectively, and the subscripts " 0 " and " $\omega$ " represent the static and dynamic components, respectively. $\hat{\rho}_{0}^{(1)}$ gives the inhomogeneity part of the background electron density $n_{\text {ih }}$ to the first order in $V_{\mathrm{sc}, 0}$, which is accurate enough for the weakly inhomogeneous electron gas. Likewise, $\hat{\rho}_{\omega}^{(1)}$ gives the excited oscillating electron density $n_{\omega}$ to the first order in $V_{\mathrm{sc}, \omega}$, while $\hat{\rho}_{\omega}^{(2)}$ gives $n_{\omega}$ to the order in $V_{\mathrm{sc}, 0} V_{\mathrm{sc}, \omega}$, which accounts for the leading-order contribution due to the ground-state inhomogeneity. We note that $\hat{\rho}_{0}$ has the property,

$$
\hat{\rho}_{0}|\mathbf{k}\rangle=f\left(E_{\mathbf{k}}\right)|\mathbf{k}\rangle,
$$

where $\langle\mathbf{r} \mid \mathbf{k}\rangle=e^{i k \mathbf{r}}$ satisfying $\hat{\mathcal{H}}_{0}|\mathbf{k}\rangle=E_{\mathbf{k}}|\mathbf{k}\rangle$ with $f\left(E_{\mathbf{k}}\right)$ being the Fermi-Dirac distribution function. Here, we take the zero temperature expression of $f\left(E_{\mathbf{k}}\right)$, i.e., $f\left(E_{\mathbf{k}}\right)=1$ for $E_{\mathbf{k}} \leqslant E_{F}$ and $f\left(E_{\mathbf{k}}\right)=0$ for $E_{\mathbf{k}}>E_{F}$. 
Taking Eq. (14) into Eq. (13) and extracting the same order terms, we get

$$
\begin{aligned}
& {\left[\hat{\mathcal{H}}_{0}, \hat{\rho}_{0}^{(1)}\right]+\left[V_{\mathrm{sc}, 0}, \hat{\rho}_{0}^{(1)}\right]=0,} \\
& {\left[\hat{\mathcal{H}}_{0}, \hat{\rho}_{\omega}^{(1)}\right]+\left[V_{\mathrm{sc}, \omega}, \hat{\rho}_{0}^{(1)}\right]=-i \hbar \omega \hat{\rho}_{\omega}^{(1)},} \\
& {\left[\hat{\mathcal{H}}_{0}, \hat{\rho}_{\omega}^{(2)}\right]+\left[V_{\mathrm{sc}, \omega}, \hat{\rho}_{0}^{(1)}\right]+\left[V_{\mathrm{sc}, 0}, \hat{\rho}_{\omega}^{(1)}\right]=-i \hbar \omega \hat{\rho}_{\omega}^{(2)} .}
\end{aligned}
$$

These equations, together with $n_{\text {ih }}=\operatorname{Tr}\left[\delta\left(x-x_{e}\right) \hat{\rho}_{0}^{(1)}\right]$ and $n_{\omega}=\operatorname{Tr}\left[\delta\left(x-x_{e}\right)\left(\hat{\rho}_{\omega}^{(1)}+\hat{\rho}_{\omega}^{(2)}\right)\right]$, lead to

$$
\begin{aligned}
n_{\mathrm{ih}}(x)= & \int d k \chi(0, k) v_{\mathrm{sc}, 0}(k) e^{i k x}, \\
n_{\omega}(x)= & \int d k \chi(\omega, k) v_{\mathrm{sc}, \omega}(k) e^{i k x} \\
& +\iint d k_{1} d k_{2} \xi\left(\omega, k_{1}, k_{2}\right) v_{\mathrm{sc}, 0}\left(k_{1}\right) v_{\mathrm{sc}, \omega}\left(k_{2}\right) e^{i\left(k_{1}+k_{2}\right) x},
\end{aligned}
$$

where

$$
\chi(\omega, k)=\frac{1}{2 \pi^{3}} \int \frac{f\left(E_{\mathbf{q}-\mathbf{k}}\right)-f\left(E_{\mathbf{k}}\right)}{\hbar \omega+E_{\mathbf{q}-\mathbf{k}}-E_{\mathbf{k}}} d \mathbf{q},
$$

relating to the RPA permittivity by $\epsilon_{m}^{R}(\omega, k)=1-$ $e^{2} \chi(\omega, k) / \epsilon_{0} k^{2}[10,11]$, and

$$
\xi\left(\omega, k_{1}, k_{2}\right)=\frac{4 k_{2}\left(k_{1}+k_{2}\right)}{\omega^{2} k_{1} m_{e}-\hbar^{2} k_{1} k_{2}^{2}\left(k_{1}+k_{2}\right)^{2}} \eta,
$$

with

$$
\eta=\chi\left(0, k_{1}\right) k_{1}+\chi\left(\omega, k_{2}\right) k_{2}-\chi\left(\omega, k_{1}+k_{2}\right)\left(k_{1}+k_{2}\right) .
$$

Expanding $\chi$ to the fourth order in $k$, we cast the expression of $n_{\omega}$ into the differential equation form, and compare the derived equation with Eq. (9) to the first order of $n_{\text {ih }}$ for being consistent with the expansion orders applied in Eq. (14). In the low-frequency regime, the derived equation agrees with Eq. (14) exactly with $\beta=\sqrt{1 / 3} v_{F}$ and $\lambda_{w}=1 / 9$. However, in the high-frequency regime, the derived equation shows a slightly different form with

$$
\frac{\partial^{2} \mathbf{J}_{1}}{\partial t^{2}}=\epsilon_{0} \omega_{p}^{2} f_{0}^{2} \frac{\partial \mathbf{E}_{1}}{\partial t}+N^{(3)} \mathbf{J}_{1}+\beta^{2}\left(\frac{d}{d x}-\frac{2 d f_{0}}{f_{0} d x}\right) Q_{1}^{\prime},
$$

where $\beta=v_{F} \sqrt{3 / 5}$ and $\lambda_{w}=1$ agreeing with the homogenous case, the new coefficient $N^{(3)}$ is

$$
N^{(3)}=v_{F}^{2}\left[\frac{1}{3 f_{0}^{2}} \frac{d^{2} f_{0}^{2}}{d x^{2}}+\frac{2}{9 k_{F}^{2} f_{0}^{2}} \frac{d^{4} f_{0}^{2}}{d x^{4}}\right],
$$

and the modified term $Q_{1}^{\prime}$ shares a similar form as $Q_{1}$ in Eq. (9) except that $N^{(0)}$ being modified to

$$
\begin{aligned}
N^{(0)}= & f_{0}^{4 / 3}+C_{0} \frac{v_{F}^{2}}{\beta^{2}}-\frac{C_{1}}{6} \frac{v_{F}^{2}}{\beta^{2}} f_{0}^{2 / 3}-\frac{C_{3}}{3} \frac{v_{F}^{2}}{\beta^{2}} f_{0}^{2} \\
& +2 N^{(2)}\left(\frac{11}{3} \frac{d^{2} f_{0}}{f_{0} d x^{2}}-\frac{13}{3}\left|\frac{d f_{0}}{d x}\right|^{2} \frac{1}{f_{0}^{2}}\right) .
\end{aligned}
$$

Here, it is worth noting that the new term $N^{(3)} \mathbf{J}_{1}$ vanishes in the homogenous case, while it contributes to Eq. (16) as the emergence of the inhomogeneity. Thus, in the surface region where the inhomogeneity is strong, such a term is expected to play an important role. Conclusively, the differences between Eqs. (16) and (9) imply the challenge for the QHT in the high-frequency regime on the one hand, and suggest possible improvements on the other hand.

\section{LINEAR-RESPONSE DYNAMICS IN THE ELECTRON-TAIL REGION}

In the electron-tail region, the electron density is negligible. For this reason, people may intuitively expect no important dynamics occurring. However, in this section, by analyzing the LRDs in the electron-tail region, we demonstrate the subtle mathematical solutions which should be treated carefully, and their important physical meaning related to the photoelectric effect.

\section{A. Solution analysis}

In this subsection, we will analyze the solutions of Eq. (9) in the electron-tail region where $f_{0} \ll 1$. Neglecting the damping, Eq. (9) under the quasistatic approximation could be rewritten as

$$
\begin{aligned}
-\omega^{2} \nabla^{2} \phi_{1}= & -\omega_{p}^{2} \nabla \cdot f_{0}^{2} \nabla \phi_{1}+\beta^{2} \nabla \cdot\left[\nabla-\frac{2 \nabla f_{0}}{f_{0}}\right], \\
& \times\left\{\left[N^{(0)}+\mathbf{N}^{(1)} \cdot \nabla-N^{(2)} \nabla^{2}\right] \nabla^{2} \phi_{1}\right\},
\end{aligned}
$$

where $\phi_{1}$ is the electric potential which relates with the electric field $\mathbf{E}_{1}$ by $\mathbf{E}_{1}=-\nabla \phi_{1}$.

For simplicity, considering the semi-infinite metal system located in $x<0$, and assuming that the system is driven by an external potential $e^{k_{\|} x+i k_{y} y+i k_{z} z}$ where $k_{\|}^{2}=k_{y}^{2}+k_{z}^{2}$. In this case, we know that $f_{0}$ in the electron-tail region is an exponentially decaying function with

$$
f_{0} \propto e^{-k_{Q} x} .
$$

This implies that $\mathbf{N}^{(1)}$ is approximately equal to $-N^{(2)} k_{Q} \hat{x}$, and the term $N^{(0)}$ is negligible comparing with the terms $\mathbf{N}^{(1)}$ and $N^{(2)}$. Then, we denote the external potential and its induced transverse potential by $\phi_{T}^{(0)}$ with

$$
\phi_{T}^{(0)}=e^{k_{\|} x}+\operatorname{Re}^{-k_{\|} x},
$$

where $R$ represents the reflection coefficient of the transverse potential, and the term $e^{i k_{y} y+i k_{z} z}$ is suppressed. Taking $\phi_{T}^{(0)}$ into Eq. (18), the associated longitudinal potential denoted as $\phi_{L}^{(0)}$ to the leading order is found to be

$$
\phi_{L}^{(0)} \simeq \frac{-2 k_{d} k_{\|} \omega_{p}^{2} f_{0}^{2}}{\omega^{2}-4 \beta^{2} N^{(2)} k_{d}^{2} k_{\|}^{2}}\left(e^{k_{\|} x}-\mathrm{Re}^{-k_{\|} x}\right) e^{-2 k_{Q} x} .
$$

Additionally, it is found that the first term and the term concerning $N^{(0)}$ on the RHS of (18) are much smaller than other terms. If we neglect these small terms, the equation will admit the longitudinal solution denoted as $\phi_{L}^{(1)}$ satisfying

$$
\beta^{2} \nabla \cdot\left(\nabla+2 k_{Q} \hat{x}\right) N^{(2)}\left(2 k_{d} \hat{x} \cdot \nabla+\nabla^{2}\right) \phi_{L}^{(1)}-\omega^{2} \phi_{L}^{(1)}=0 .
$$

Assuming that $\phi_{L}^{(1)}$ has the exponential solution $e^{\mathrm{i} k_{\mathrm{Lx}} x+\mathrm{i} k_{\mathrm{y}} y+\mathrm{i} k_{\mathrm{z}} z}$, we derive that

$$
k_{\mathrm{Lx}}=i k_{Q} \pm i \sqrt{k_{\|}^{2}+k_{Q}^{2} \pm \frac{\omega}{\beta \sqrt{N^{(2)}}}} .
$$


$\phi_{L}^{(1)}$ have four independent solutions, whose important indications will be discussed in Sec. VII B.

$\phi_{L}^{(0)}$ and $\phi_{L}^{(1)}$ determine the induced electron density $n_{1}$ in the tail region. We can split $n_{1}$ into

$$
n_{1}=n_{1}^{(0)}+n_{1}^{(1)}
$$

where $n_{1}^{(0)}$ and $n_{1}^{(1)}$ correspond to $\phi_{L}^{(0)}$ and $\phi_{L}^{(1)}$, respectively. The expression for $\phi_{L}^{(0)}$ results in

$$
n_{1}^{(0)} \propto e^{-2 k_{Q} x+k_{\|} x}-R e^{-2 k_{Q} x-k_{\|} x} .
$$

For $n_{1}^{(1)}$, its behavior is simply determined by the four solutions of $k_{\mathrm{Lx}}$ in Eq. (21).

\section{B. Selection of physical solutions}

In the QHT, we are faced by the challenge of having in general four solutions as compared to only two in the TFHT. This suggests that not all of the four solutions may be physically relevant. In order to analyze $k_{\mathrm{Lx}}$ in Eq. (21) and select the physical ones, we denote the four solutions of $k_{\mathrm{Lx}}$ as

$$
\begin{aligned}
& k_{\mathrm{Lx}}^{(1)}=i k_{Q}-i \sqrt{k_{\|}^{2}+k_{Q}^{2}+\omega / \beta \sqrt{N^{(2)}}}, \\
& k_{\mathrm{Lx}}^{(2)}=i k_{Q}+i \sqrt{k_{\|}^{2}+k_{Q}^{2}+\omega / \beta \sqrt{N^{(2)}}}, \\
& k_{\mathrm{Lx}}^{(3)}=i k_{Q}-i \sqrt{k_{\|}^{2}+k_{Q}^{2}-\omega / \beta \sqrt{N^{(2)}}}, \\
& k_{\mathrm{Lx}}^{(4)}=i k_{Q}+i \sqrt{k_{\|}^{2}+k_{Q}^{2}-\omega / \beta \sqrt{N^{(2)}}},
\end{aligned}
$$

where the square-root operator is defined with $\sqrt{1}=1$ and $\sqrt{-1}=-i$. Further, we define an important quantity $\omega_{c}^{Q}$ with

$$
\omega_{c}^{Q}=\frac{\hbar\left(k_{Q}^{2}+k_{\|}^{2}\right) \sqrt{\lambda_{w}}}{2 m_{e}},
$$

which leads to $k_{\|}^{2}+k_{Q}^{2}-\omega_{c}^{Q} / \beta \sqrt{N^{(2)}}=0$. In Sec. VIID, it will be demonstrated that $\omega_{c}^{Q}$ just defines the threshold frequency of the photoelectric effect.

To select the physical solutions among $k_{\mathrm{Lx}}^{(i)}(i=1,2,3,4)$, we take the special case $k_{\|}=0$ as an example. In this case, when $\omega<\omega_{c}^{Q}, k_{\mathrm{Lx}}^{(i)}$ are all imaginary numbers. In particular, $k_{\mathrm{Lx}}^{(1)}$ has $\operatorname{Im}\left[k_{L x}^{(1)}\right]<0$ representing the amplifying solution. For a passive (or lossy) medium, the electron density cannot amplify, and accordingly $k_{\mathrm{Lx}}^{(1)}$ is not physical. The other three formal solutions all represent decaying solutions with $\operatorname{Im}\left[k_{L x}^{(2)}\right]>2 k_{Q}, \operatorname{Im}\left[k_{L x}^{(3)}\right]<k_{Q}$, and $k_{Q}<\operatorname{Im}\left[k_{l x}^{(4)}\right]<2 k_{Q}$. To analyze these decaying solutions, we borrow insights from quantum mechanics. The linear-response theory of quantum mechanics tells us that

$$
n_{1} \propto \psi_{0}^{*} \psi_{1}+\psi_{0} \psi_{1}^{*},
$$

where $\psi_{0}$ represents the ground-state wave function with $\psi_{0} \propto \sqrt{n_{0}} \propto e^{-k_{Q} x}$ qualitatively, and $\psi_{1}$ represents the excited state wave function with $\psi_{1} \propto e^{-k_{e} x}$. Since the ground state with less energy should be more bounded than the excited state, we deduce that $\operatorname{Re}\left(k_{e}\right)<k_{Q}$. Thus, there is $n_{1} \propto e^{-\alpha x}$ with $k_{Q} \leqslant \operatorname{Im}(\alpha)>2 k_{Q}$. This leads to the conclusion that the mode $k_{\mathrm{Lx}}^{(3)}$ is not physical since $\operatorname{Im}\left[k_{L x}^{(3)}\right]<k_{Q}$, while the modes $k_{\mathrm{Lx}}^{(2,4)}$ are physical with the one $k_{\mathrm{Lx}}^{(4)}$ being dominant. When $\omega>\omega_{c}^{Q}, k_{\mathrm{Lx}}^{(1)}$ and $k_{\mathrm{Lx}}^{(2)}$ keep the same characteristics as the case $\omega<\omega_{c}^{Q}$. However, $k_{\mathrm{Lx}}^{(3,4)}$ become complex numbers with $\operatorname{Re}\left[k_{\mathrm{Lx}}^{(3)}\right]<0$ and $\operatorname{Re}\left[k_{\mathrm{Lx}}^{(4)}\right]>0$. The complex valued $k_{\mathrm{Lx}}$ indicates the propagating characteristics of the excited state. Respecting the causality relation that the excited propagating wave can only be outgoing, the mode $k_{\mathrm{Lx}}^{(4)}$ is justified to be physical, not the mode $k_{\mathrm{Lx}}^{(3)}$. Conclusively, in the whole frequency range, the modes $k_{\mathrm{Lx}}^{(2,4)}$ are the physical ones. For $k_{\|} \neq 0$, the same conclusion can be drawn following similar arguments.

\section{Interpretation of physical solutions}

In the above subsection, we conclude that the modes $k_{\mathrm{Lx}}^{(2,4)}$ are the physically relevant ones among the four formal mathematical solutions. Here, we will further justify this conclusion seriously, and give an unambiguous interpretation of the physical modes based on microscopic quantum analysis. Considering the semi-infinite metal system located in $x<0$, and driving this system by an external electric potential $V_{\text {ext }}(x) \exp \left(i k_{\|} y-i \omega t\right)$, the linearly excited electron density $n_{1}$ within the framework of the Kohn-Sham orbitals is

$$
n_{1}=n_{1}^{+}+n_{1}^{-}
$$

where

$$
\begin{aligned}
& n_{1}^{+}=\frac{1}{\pi^{3}} \iint_{k_{1}^{2}+k_{2}^{2}<k_{F}^{2}} d k_{1} d k_{2} \sqrt{k_{F}^{2}-k_{1}^{2}-k_{2}^{2}} \psi_{k_{1}}^{*} \psi_{k_{1} k_{2}}^{+}, \\
& n_{1}^{-}=\frac{1}{\pi^{3}} \iint_{k_{1}^{2}+k_{2}^{2}<k_{F}^{2},} d k_{1} d k_{2} \sqrt{k_{F}^{2}-k_{1}^{2}-k_{2}^{2}} \psi_{k_{1}}^{0} \psi_{k_{1} k_{2}}^{-*} .
\end{aligned}
$$

$\psi_{k}$ represents the electron wave functon in the absence of the perturbation satisfying

$$
\left[-\frac{\hbar^{2}}{2 m_{e}} \frac{d^{2}}{d x^{2}}+V_{\mathrm{eff}}(x)\right] \psi_{k}(x)=\hbar \omega_{k} \psi_{k}(x),
$$

where $V_{\text {eff }}$ represents the effective potential which a single electron experiences [15,55]. $\psi_{k_{1} k_{2}}^{ \pm}$represent the linear order corrections to $\psi_{k_{1}}$, and are expressed by

$$
\begin{aligned}
& \psi_{k_{1} k_{2}}^{+}(x)=\int d x^{\prime} G_{k_{1} k_{2}}^{+}\left(x, x^{\prime}\right) \psi_{k_{1}}\left(x^{\prime}\right) V_{\mathrm{scf}}\left(x^{\prime}\right), \\
& \psi_{k_{1} k_{2}}^{-}(x)=\int d x^{\prime} G_{k_{1} k_{2}}^{-}\left(x, x^{\prime}\right) \psi_{k_{1}}\left(x^{\prime}\right) V_{\mathrm{scf}}^{*}\left(x^{\prime}\right),
\end{aligned}
$$

where $V_{\text {scf }}$ is the self-consistent potential due to the external potential. Furthermore, $G_{k_{1} k_{2}}^{ \pm}$are the single-particle Green's functions satisfying

$$
\left[-\frac{\hbar^{2}}{2 m_{e}} \frac{d^{2}}{d x^{2}}+V_{\mathrm{eff}}(x)+U_{\mathrm{eff}}^{ \pm}\right] G_{k_{1} k_{2}}^{+}\left(x, x^{\prime}\right)=-\delta\left(x-x^{\prime}\right),
$$

where

$$
U_{\mathrm{eff}}^{ \pm}=-\hbar\left(\omega_{k_{1}} \pm \omega_{0}\right)+\frac{\hbar^{2} k_{\|}^{2}+2 \hbar^{2} k_{\|} k_{2}}{2 m_{e}} .
$$

Observing the expressions of $n_{1}^{ \pm}$, we find that $n_{1}^{ \pm}$in the electron-tail region are mainly contributed by their Fourier 
components at $k_{1}=k_{F}$, which are denoted as $n_{1, k_{F}}^{ \pm}$. Further, it is found that

$$
n_{1, k_{F}}^{ \pm} \propto e^{i k_{\mathrm{Lx}}^{ \pm} x}
$$

where

$$
k_{\mathrm{Lx}}^{ \pm}=i k_{D}+i \sqrt{k_{\|}^{2}+k_{D}^{2} \mp \frac{2 m_{e} \omega}{\hbar}},
$$

with $k_{D}$ relates to the DFT work function $W_{F}^{D}$ by $W_{F}^{D}=$ $\hbar^{2} k_{D}^{2} / 2 m_{e}$. Interestingly, the expressions of $k_{\mathrm{Lx}}^{ \pm}$share the similar forms as $k_{\mathrm{Lx}}^{(2,4)}$ with $k_{\mathrm{Lx}}^{(4)}$ corresponding to $k_{\mathrm{Lx}}^{+}$, and $k_{\mathrm{Lx}}^{(2)}$ corresponding to $k_{\mathrm{Lx}}^{-}$. In this sense, the longitudinal modes $k_{\mathrm{Lx}}^{(4,2)}$ just characterize the induced electron density $n_{1}^{ \pm}$, respectively. Furthermore, we notice that $k_{\mathrm{Lx}}^{(2,4)}$ have exactly the same form as $k_{\mathrm{Lx}}^{ \pm}$if $\lambda_{w}=1$.

\section{Interpretation of $\omega_{c}^{Q}$}

The above discussions show that the physical solution $k_{\mathrm{Lx}}^{(4)}$ is complex valued when $\omega>\omega_{c}^{Q}$, which implies that the bounded electron being excited to the propagating one by absorbing the photon energy, i.e., the photoelectric effect. Based on such understanding, $\omega_{c}^{Q}$ just physically represents the threshold frequency of the photoelectric effect for different $k_{\|}$. When $k_{\|}=0, \omega_{c}^{Q}$ achieves the minimal value, which just corresponds to the work function. However, unless $\lambda_{w}=1$, the work function defined in this way usually differs with $W_{F}^{Q}$ defined in Eq. (8) as clearly seen from their relation,

$$
W_{F}^{Q}=\sqrt{\lambda_{w}} \hbar \omega_{c}^{Q}\left(k_{\|}=0\right)
$$

From this perspective, $\lambda_{w} \rightarrow 1$ in the electron-tail region can reconcile the inconsistence of the threshold frequency of the photoelectric effect and the work function. Thus, the fact suggests that $\lambda_{w} \rightarrow 1$ in the electron-tail region consistent with the same conclusion in Sec. III B.

\section{REMARK ON SECS. V-VII}

The findings in Secs. V-VII point out several possible improvements for the QHT. In particular, we show that the parameter $\lambda_{w}$ should depend on both frequency and space, and a new term appears in the linear-response equation when the inhomogeneity of the ground electron density becomes important in the high-frequency regime $\omega \gg \gamma$. Additionally, the important physical indications of the QHT, such as capturing the bulk plasmon modes and also the photoelectric effect, are demonstrated. However, it is still challenging to build a HT fully consistent with these findings due to our limited knowledge in the surface region. At this stage, we refrain explorations in this direction, leaving them for future work. Instead, we will focus on the details of the simple QHT, i.e., Eq. (9) with $\beta=\sqrt{1 / 3} v_{F}$ and $\lambda_{w}$ being a constant, by numerical analysis, and demonstrate both the advantages and disadvantages of such a theory. It is worth noting that the general findings in the following sections do not depend on specific values of $\beta$ and $\lambda_{w}$.

\section{BOUNDARY CONDITIONS FOR LINEAR-RESPONSE DYNAMICS}

For solving the LRD, correct boundary conditions (BCs) are of essential importance. One might speculate that it is sufficient to assume a simple BC where the excited electron density $n_{1}$ is truncated (set to zero) deep into the electron-tail region. However, care should be taken since $n_{0}$ would approach zero as well. First, the knowledge in Sec. VII B tells us that the simple BC fails to filter out the unphysical mode $k_{\mathrm{Lx}}^{(3)}$. Second, such BC fails to define a unique solution. To illustrate the second point clearly, we consider a specific case of a metallic slab driven by a uniform electric field. At each side of the slab, we know that the simple BC allows four unknown quantities corresponding to the three longitudinal modes $k_{\mathrm{Lx}}^{(2,3,4)}$ and also the induced transverse potential, which are demonstrated in Sec. VII A. There are in total eight unknown quantities. To determine these quantities, one can evolve Eq. (18) from one side of the slab to the other side for an example by some numerical integration scheme, and glue these unknowns. Accordingly, one can obtain six equations since Eq. (18) is a sixth-order differential equation. Six equations are of course insufficient to determine eight quantities. Thus, the simple BC allows multiple solutions, which is numerically confirmed in Appendix B.

To demonstrate the correct $\mathrm{BC}$, we define a new quantity $n_{1}^{\prime}$ with

$$
n_{1}^{\prime}=\frac{n_{1}}{\sqrt{n_{0}}} .
$$

As shown in the following, the modifed BC $n_{1}^{\prime} \rightarrow 0$ in the electron-tail region defines the problem well. When $\omega<\omega_{c}^{Q}$, it is obvious that the modified BC filters out the unphysical modes $k_{\mathrm{Lx}}^{(1,3)}$ automatically. When $\omega>\omega_{c}^{Q}$, at first glance, it seems that the modified $\mathrm{BC}$ becomes invalid, since the modes $k_{\mathrm{Lx}}^{(3,4)}$ have the same exponential decaying rate as $\sqrt{n_{0}}$. However, this could be easily overcome by adding a small damping $\gamma$ into the system which makes the mode $k_{\mathrm{Lx}}^{(3)}$ decay slower than $\sqrt{n_{0}}$ and the mode $k_{\mathrm{Lx}}^{(4)}$ decay faster than $\sqrt{n_{0}}$. Then, the mode $k_{\mathrm{Lx}}^{(3)}$ is effectively filtered out. So far, we have proved that the modified BC is physically sound. Next, following the same mathematical argument as in the above paragraph, it is easy to show that the modified BC determines six unknown quantities by six equations, i.e., a problem with a unique solution.

To conclude this section, the case of the semi-infinite metal system is discussed. In this case, one also needs the BC in the bulk region. Below the plasma frequency, the QHT admits four longitudinal modes among which two modes decay away from the metal surface, and the other two amplify. Clearly, the modified $\mathrm{BC} n_{1}^{\prime} \rightarrow 0$ in the deep bulk region can filter out the amplifying modes. Above the plasma frequency, there are two plasmon modes propagating in the opposite directions. The one propagating away from the surface is the physical one, which can also be selected by the modified BC with a finite damping. Thus, the modified $\mathrm{BC}$ is also adaptable to the semi-infinite metal system. 


\section{NUMERICAL RESULTS FOR LINEAR-RESPONSE DYNAMICS}

In this section, we will investigate the details of the QHT for characterizing the LRD by numerical analysis. We again focus on the system of the semi-infinite metal located in $x<0$. For the QHT, the response is calculated with the FEM also employed in our numerical analysis of the ground-state electron density above. For comparisons, we also implement the time-dependent local-density approximation (TDLDA) method [78-80]. The numerical computation of the LRD by the QHT is much more efficient than the TDLDA, because the heavy computation of the nonlocal response function in the TDLDA is greatly reduced by the hydrodynamic differential equation.

\section{A. Distribution of induced charge}

We consider the simple metal with $r_{s}=4$ driven by a uniform electric field in the $x$ direction. For the QHT, we set $\lambda_{w}=0.5$ as in Fig. 1(a). The excited electron density $n_{1}$ at $\omega=0.2 \omega_{p}$ and $0.8 \omega_{p}$ are plotted in Fig. 3(a). $\operatorname{Re}\left(n_{1}\right)$ is in phase with the driven field, while $\operatorname{Im}\left(n_{1}\right)$ is out of phase with the driving field as a manifestation of the damping. In the QHT, $n_{1}$ shows a diffusive profile as the TDLDA, however the oscillation feature in the bulk region is nearly invisible owing to the exponential decay as discussed in Sec. V. For the lossless case $(\gamma=0)$ in the QHT, we observed that $\operatorname{Im}\left(n_{1}\right)$ is zero at $\omega=0.2 \omega_{p}$ and becomes nonzero at $\omega=0.8 \omega_{p}$. This is due to the bounded electron being excited into the propagating state when $\omega>\omega_{c}^{Q}$, with $\omega_{c}^{Q}=0.74 \omega_{p}$ in this case. Increasing $\gamma$ to $0.025 \omega_{p}$, thereby accounting for the damping channels not included in the QHT such as the electron-hole excitations, $\operatorname{Im}\left(n_{1}\right)$ becomes nonzero at $\omega=0.2 \omega_{p}$.

Finally, we consider the simple metal with $r_{s}=2$. For the QHT, we choose $\lambda_{w}=0.25$ as in Fig. 1(a). The results are plotted in Fig. 3(b) which shows the overall similar behavior as in Fig. 3(a).

\section{B. Centroid of induced charge}

To make a more quantitative judgment on the accuracy of the QHT, we discuss the centroid of the induced charge [81], which is denoted as $d_{c}$ and defined by

$$
d_{c}(\omega)=\frac{\int d x \times n_{1}(\omega, x)}{\int d x n_{1}(\omega, x)} .
$$

$\operatorname{Re}\left(d_{c}\right)$ can be positive or negative meaning that the induced charge is effectively located outside or inside the metal, respectively. $\operatorname{Im}\left(d_{c}\right)$ captures the strength of surface damping of the system. Such a parameter has a determinative role on the dispersion of the surface plasmon (SP) resonance. For three typical geometries of the metal system, i.e., plane interface, cylinder, and sphere, we summarize the dispersion expressions of the SP resonance in Table III $[45,50,78,81,82]$. Clearly, $\operatorname{Re}\left(d_{c}\right)>0$ leads to a redshift of the SP resonance with the SP wave number increasing or the size decreasing, while $\operatorname{Re}\left(d_{c}\right)<0$ consequently leads to a blueshift. Quite intuitively, $\operatorname{Im}\left(d_{c}\right)$ is linked to a resonance broadening.

In Fig. 4(a), we plot $d_{c}$ for the simple metal with $r_{s}=4$. The important frequencies $\omega_{c}^{D, Q}$ and $\omega_{\mathrm{mp}}^{D, Q}$ are indicated
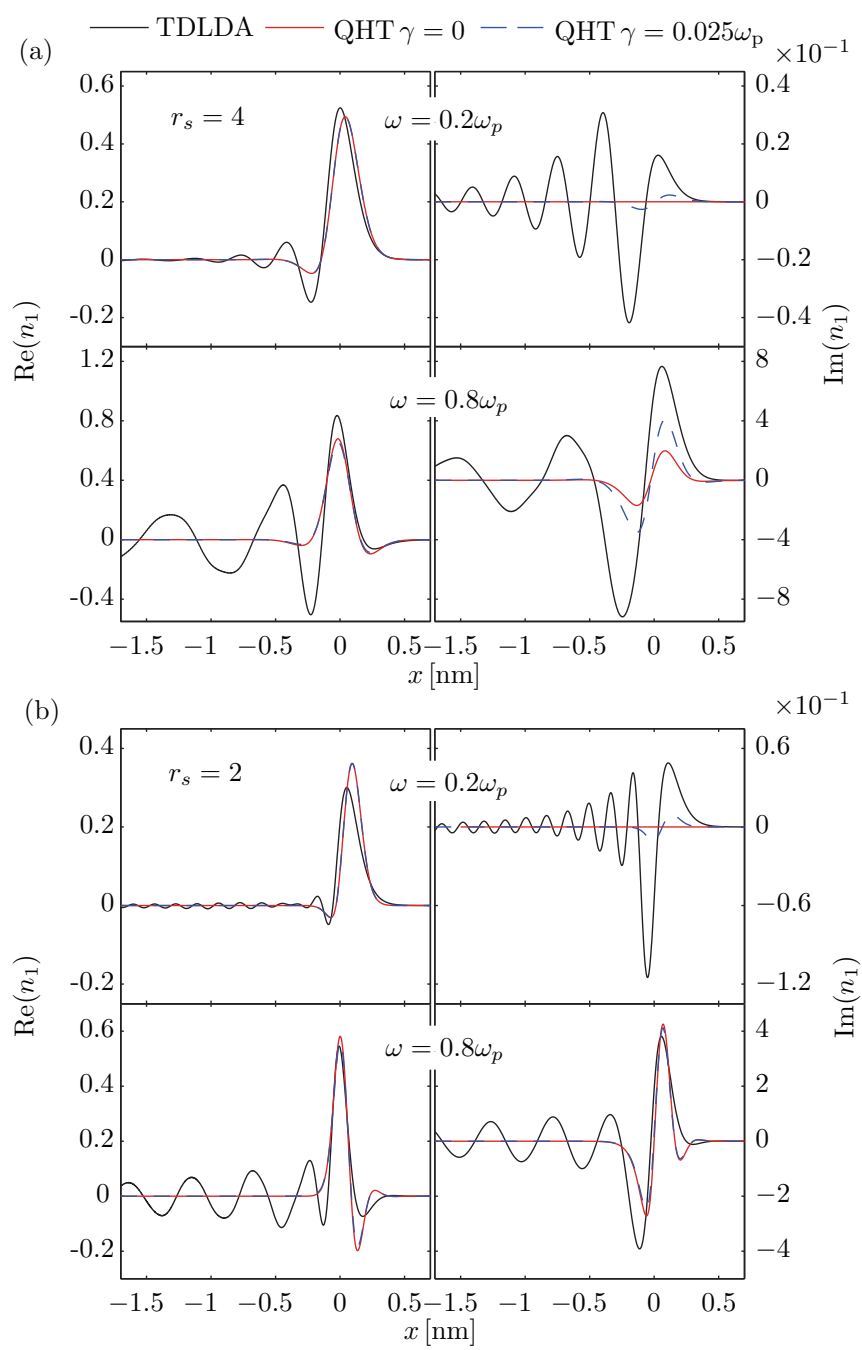

FIG. 3. (Color online) Excited electron density of the semiinfinite metal structure driven by a uniform electric field in the $x$ direction for the simple metals with (a) $r_{s}=4$ and (b) $r_{s}=2$. The metal is in located in the region $x<0$. The results are calculated by the QHT and TDLDA.

by arrows, and the superscripts " $D$ " and " $Q$ " indicate the TDLDA and QHT values, respectively. $\omega_{c}$ represents the threshold frequency of the photoelectric effect as introduced in Sec. VIID. We know that $W_{F}^{D}=\hbar \omega_{c}^{D}$ for the TDLDA, and $W_{F}^{Q}=\sqrt{\lambda_{w}} \hbar \omega_{c}^{Q}$ for the QHT. Above $\omega_{c}, \operatorname{Im}\left(d_{c}\right)$ shows a slight enhancement owing to the excitation of the propagating

TABLE III. SP resonance dispersion for plane interface, cylinder, and sphere shaped metallic structures. $q$ represents the SP wave number for the plane interface, $l$ represents the angular momentum number of the cylindrical or spherical SP harmonics, and $r$ represents the radius of the cylinder or sphere.

\begin{tabular}{lc}
\hline \hline Geometry & SP resonance \\
\hline Plane interface & $\frac{\omega_{p}}{\sqrt{2}}\left(1-\frac{q d_{c}}{2}\right)+\mathcal{O}\left(q^{2} d_{c}^{2}\right)$ \\
Cylinder & $\frac{\omega_{p}}{\sqrt{2}}\left(1-\frac{l d_{c}}{2 r}\right)+\mathcal{O}\left(\frac{l^{2} d_{c}^{2}}{r^{2}}\right)$ \\
Sphere & $\omega_{p} \sqrt{\frac{l}{l+1}}\left(1-\frac{(l+1) d_{c}}{2 r}\right)+\mathcal{O}\left(\frac{l^{2} d_{c}^{2}}{r^{2}}\right)$ \\
\hline \hline
\end{tabular}



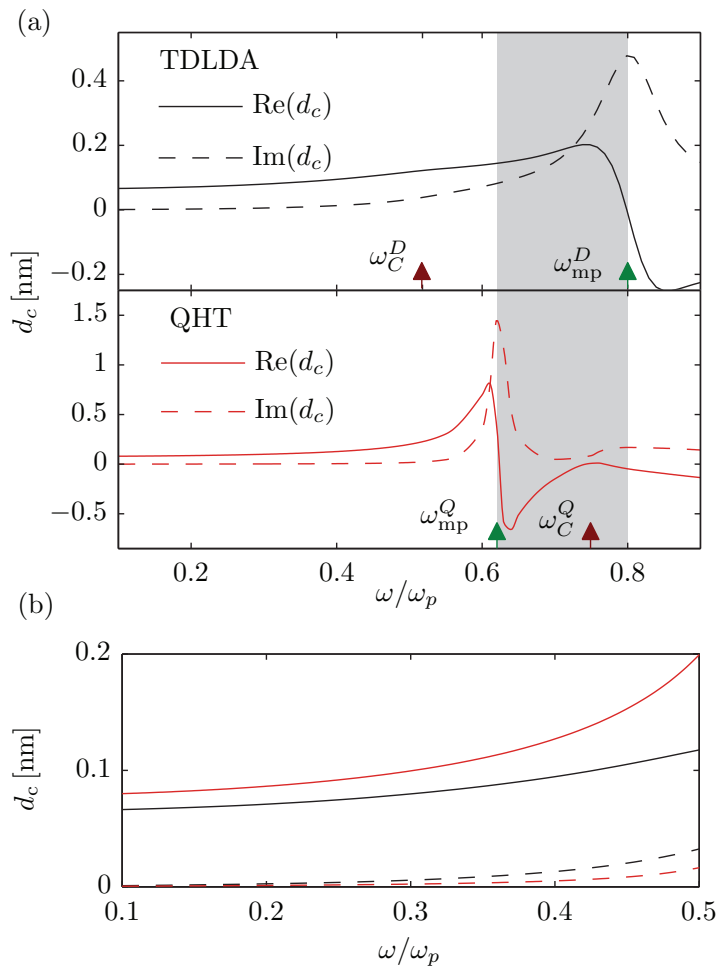

FIG. 4. (Color online) Centroid of the induced charge for the semi-infinite simple metal structure with $r_{s}=4$. The results calculated by the TDLDA and QHT. For the QHT, we choose $\lambda_{w}=0.5$ and $\gamma=0.025 \omega_{p}$. The arrow markers $\omega_{c}^{D, Q}$ and $\omega_{\mathrm{mp}}^{D, Q}$ indicate the threshold frequency of the photoelectric effect and the multipole SP resonance frequency, respectively, where the superscripts " $D$ " and " $Q$ " indicate the TDLDA and QHT values, respectively. The shaded region covers the frequency range between $\omega_{\mathrm{mp}}^{Q}$ and $\omega_{\mathrm{mp}}^{D}$, where the values of $\operatorname{Re}\left(d_{c}\right)$ for the TDLDA and QHT show opposite signs.

state. $\omega_{\mathrm{mp}}$ represents the resonance frequency of $d_{c}$. Across $\omega_{\mathrm{mp}}, \operatorname{Re}\left(d_{c}\right)$ experiences an abrupt change from the positive to the negative value, while $\operatorname{Im}\left(d_{c}\right)$ shows a peak. Such behavior physically corresponds to the multipole (MP) surface-plasmon resonance. At resonance, the dipolelike charge distribution is established in the surface region, and the induced electric field acts as the restoring force to drive the resonance. To illustrate this picture clearly, the charge distributions around $\omega_{\mathrm{mp}}$ for the TDLDA and QHT are plotted in Fig. 5, where the dipolelike charge distributions are clearly observed.

Even though the QHT successfully predicts the existence of $\omega_{c}$ and $\omega_{\mathrm{mp}}$, it gives the relation,

$$
\omega_{c}^{Q}>\omega_{\mathrm{mp}}^{Q}
$$

contradicting with the TDLDA one $\omega_{c}^{D}<\omega_{\mathrm{mp}}^{D}$. The wrong relation is a universal property of the QHT independent of $\beta$ and $\lambda_{w}$ as proven in Appendix B, and reflects the intrinsic shortcoming of the theory.

The frequency region between $\omega_{\mathrm{mp}}^{Q}$ and $\omega_{\mathrm{mp}}^{D}$ is highlighted (grayscale) in Fig. 4(a). In this region, the QHT gives the opposite sign of $\operatorname{Re}\left(d_{c}\right)$ compared with the TDLDA, and accordingly predicts the wrong trend of the SP dispersion if the SP frequency is inside this region. For convenience, we

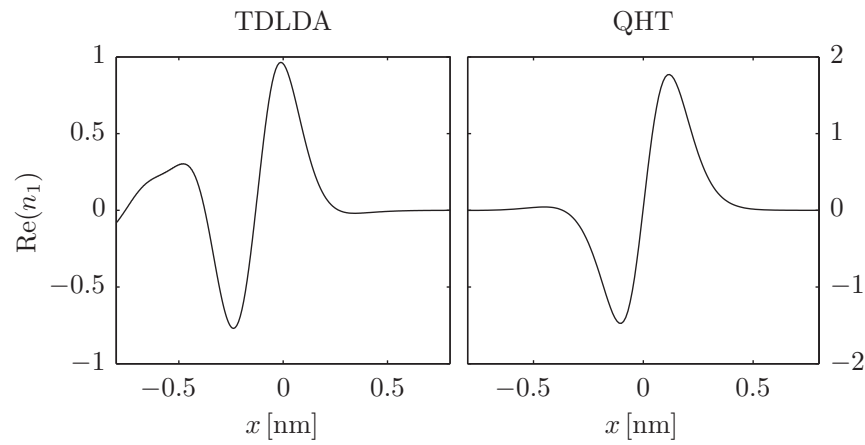

FIG. 5. The induced charge for the semi-infinite simple metal structure with $r_{s}=4$ around $\omega_{\mathrm{mp}}$. The results are calculated by the TDLDA at $\omega=\omega_{\mathrm{mp}}^{D}-0.02 \omega_{p}$ and QHT at $\omega=\omega_{\mathrm{mp}}^{Q}-0.01 \omega_{p}$.

name the frequency region,

$$
\omega \in\left(\omega_{\mathrm{mp}}^{Q}, \omega_{\mathrm{mp}}^{L}\right),
$$

as the opposite centroid $(O C)$ frequency region. Away from the OC frequency region, especially for $\omega \ll \omega_{\mathrm{mp}}^{Q}$, the values of $d_{c}$ of the QHT and TDLDA have a reasonable agreement as observed in Fig. 4(b). Here, it is worth noting that the frequency range in Fig. 4(b) is much larger than the damping frequency $\gamma$. This indicates that the QHT under the LEA approximation can still be able to predict the LRD qualitatively well in the high-frequency regime.

Next, we consider the simple metal with $r_{s}=2$, and plot $d_{c}$ in Fig. 6. As in Fig. 4, the QHT gives the wrong relation between $\omega_{c}^{Q}$ and $\omega_{\mathrm{mp}}^{Q}$ confirming the universal relation of Eq. (28). Additionally, the OC frequency region in this case is much wider than in Fig. 4.

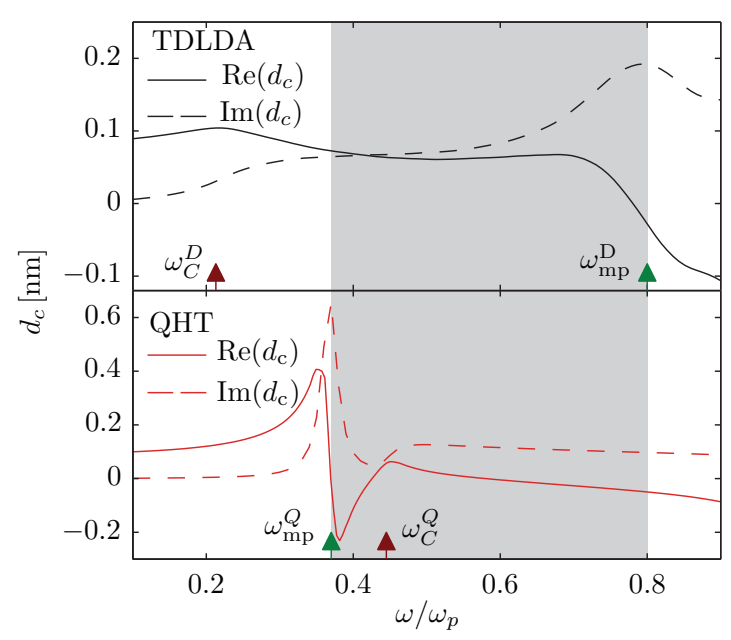

FIG. 6. (Color online) Centroid of the induced charge $d_{c}$ for the semi-infinite simple metal structure with $r_{s}=2$. The results calculated by the TDLDA and QHT. For the QHT, we choose $\lambda_{w}=$ 0.25 and $\gamma=0.025 \omega_{p}$. The arrow markers $\omega_{c}^{D, Q}$ and $\omega_{\mathrm{mp}}^{D, Q}$ indicate the threshold frequency of the photoelectric effect and the multipole SP resonance frequency, respectively, where the superscripts " $D$ " and " $Q$ " indicate the TDLDA and QHT values, respectively. The shaded region covers the frequency range between $\omega_{\mathrm{mp}}^{Q}$ and $\omega_{\mathrm{mp}}^{D}$, where the values of $\operatorname{Re}\left(d_{c}\right)$ for the TDLDA and QHT show opposite signs. 


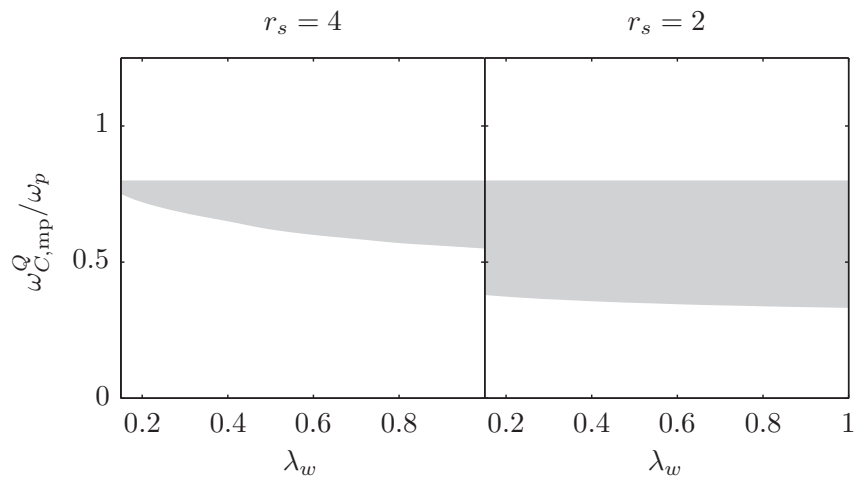

FIG. 7. The OC frequency region (shadow areas) as a function of $\lambda_{w}$ for the simple metals with $r_{s}=4,2$.

\section{OC Frequency region}

Obviously, it is desirable to have a narrower OC frequency region for the QHT, i.e., more agreement between $\omega_{\mathrm{mp}}^{Q}$ and $\omega_{\mathrm{mp}}^{L}$. Accordingly, this calls for the optimization of $\lambda_{w}$. Here, we calculate the OC frequency region as a function of $\lambda_{w}$, and plot the results in Fig. 7. Two observations are worth noting: (1) the OC frequency range shrinks as $\lambda_{w}$ decreases, indicating that smaller $\lambda_{w}$ is better than a larger one; (2) the OC frequency range is much wider for the metal with $r_{s}=2$ than with $r_{s}=4$ independent of the choice of $\lambda_{w}$.

To further illustrate how different values of $\lambda_{w}$ and resulted OC frequency regions affect the LRD, we choose $\lambda_{w}=$ $0.5,0.15$ and $\gamma=0.025 \omega_{p}$, and calculate the dispersions of the SP and MP resonances for the simple metal with $r_{s}=4$. The results are illustrated in Fig. 8. The TDLDA results show that the SP resonance starting from $\omega_{p} / \sqrt{2}$ experiences a redshift initially as the wave number increases, and then turns to blueshift after the wave number exceeds $0.14 k_{F}$. The redshift for the small wave number is also captured by the QHT with $\lambda_{w}=0.15$, but not with $\lambda_{w}=0.5$. This could be understood from Fig. 7 that the frequency $\omega_{p} / \sqrt{2}$ is outside the $\mathrm{OC}$ frequency region for $\lambda_{w}=0.15$, while it is inside for $\lambda_{w}=0.5$. Additionally, for the large wave number of the SP mode, the QHT and TDLDA show an

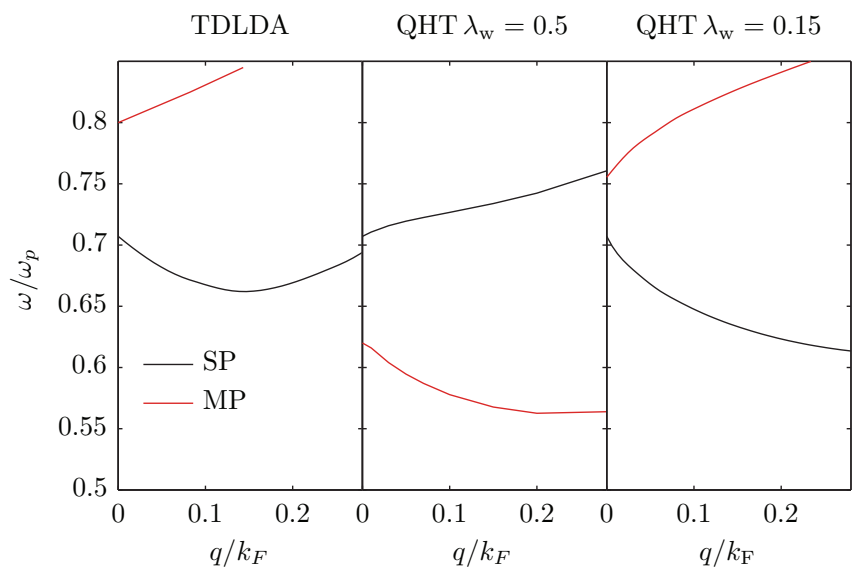

FIG. 8. (Color online) The dispersion of the surface plasmon and multipole surface plasmon resonances for the simple metal with $r_{s}=$ 4. The results are calculated by the TDLDA and QHT. increased disagreement. Next, for the MP resonance, the TDLDA result indicates that the resonance shows blueshift as the wave number increasing, which again agrees with the QHT $\lambda_{w}=0.15$ qualitatively. The above numerical results suggest that $\lambda_{w}=0.15$ is superior to $\lambda_{w}=0.5$ for characterizing the LRD. However, as demonstrated in Sec. III, we know that $\lambda_{w}=0.5$ is better for the ground state instead. Such inconsistency manifests the limitation of the QHT, and also suggests the trade-off of $\lambda_{w}$ for characterizing both the ground state and the LRD.

\section{CONCLUSIONS AND DISCUSSIONS}

In conclusion, we investigate the quantum-hydrodynamic theory (QHT) for linear-response dynamics (LRD) of metallic media, offering a thorough comparison of QHT with microscopic quantum theory.

We demonstrate that the QHT can predict the following important quantities: (1) the work function $W_{F}^{Q}$ from the ground state electron density; (2) the threshold frequency of the photoelectric effect $\omega_{c}^{Q}$ from the analysis of the LRD in the electron-tail region; (3) the mulipole resonance $\omega_{\mathrm{mp}}^{Q}$; (4) the correct trend of frequency shifts of both SP and MP resonances by choosing a proper parameter $\lambda_{w}$. Even in light of the above appealing advantages, the QHT has the following intrinsic limitations: (1) The threshold frequency of the photoelectric effect is not consistent with the work function; (2) the incorrect relation between the multipole surface plasmon resonance and the threshold frequency of the photoelectric effect.

For numerical solutions of the LRD within the QHT, we define the nontrivial boundary condition. From the numerical analysis, we also explore the centroid of induced charge, identifying an important frequency region where the QHT fails to predict the correct sign of the centroid of the induced charge. Such frequency region can be reduced by choosing a smaller $\lambda_{w}$.

We also show some possibilities for improving the QHT.

(1) $\lambda_{w}$ parameter. In the bulk region, $\lambda_{w}$ depends on frequency with $\lambda_{w}=1 / 9$ for $\omega \ll \gamma$ and $\lambda_{w}=1$ for $\omega \gg \gamma$. In the electron-tail region, we suggest $\lambda_{w} \rightarrow 1$ to make the threshold frequency of the photoelectric effect consistent with the work function.

(2) New and modified terms. By analyzing the LRD in a weakly inhomogeneous electron gas, it is found that, in the high-frequency regime where $\omega \gg \gamma$, a new term $N^{(3)} \mathbf{J}_{1}$ should be added, and a slight modification to the coefficient $N^{(0)}$ should be taken into account. In our future work, we aim to improve the QHT for being consistent with the above findings.

\section{ACKNOWLEDGMENTS}

The Center for Nanostructured Graphene is sponsored by the Danish National Research Foundation, Project No. DNRF58. The work was also supported by the Danish Council for Independent Research-Natural Sciences, Project No. 1323-00087. We also acknowledge financial support from the Lundbeck Foundation, Grant No. 70802. We thank Professor N. Asger Mortensen, Associate Professor Martijn Wubs, Thomas Christensen, Dr. Weihua Wang, Dr. Pu Zhang, and Dr. Giuseppe Toscano for many fruitful discussions, manuscript reading, and suggestions. 


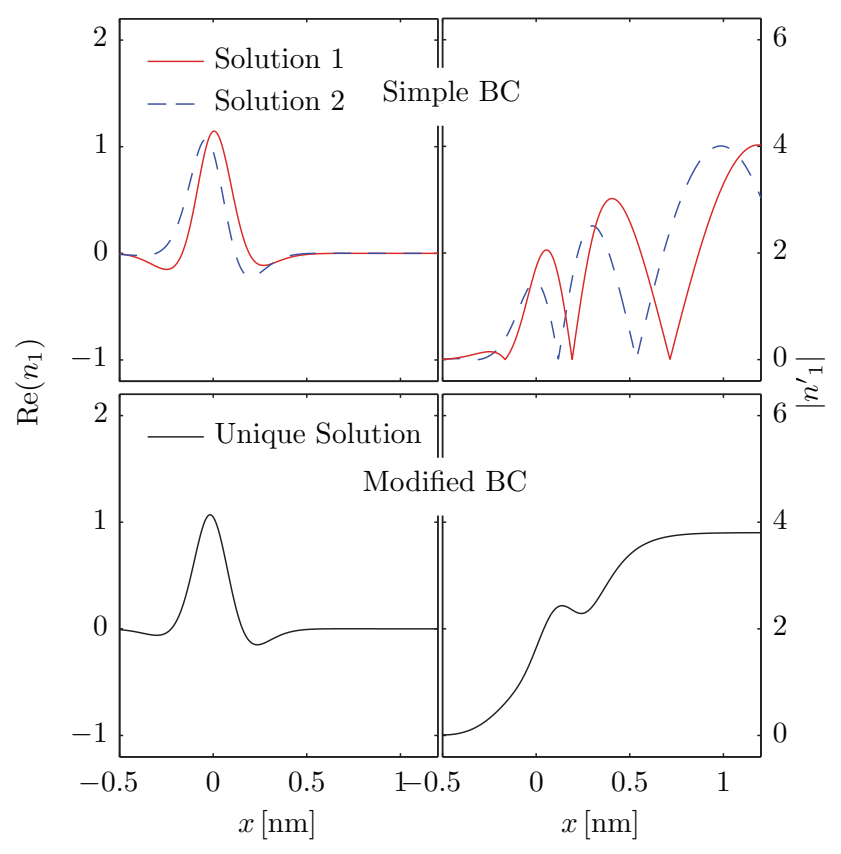

FIG. 9. (Color online) $n_{1}$ and $n_{1}^{\prime}$ calculated by the simple and modified BCs for the semi-infinite metal structure with $r_{s}=4$. The system is driven by a uniform electric field in the $x$ direction at $\omega=0.8 \omega_{p}$, and $\lambda_{w}=0.5$ for the QHT.

\section{APPENDIX A: BOUNDARY CONDITION}

In this section, we numerically prove the following: The simple BC that the induced electron density $n_{1}$ approaches zero in the electron-tail region is not correct and gives multiple solutions, while the modified BC that the defined quantity $n_{1}^{\prime}=$ $n_{1} / \sqrt{n_{0}}$ approaches zero in the electron-tail region determines the unique physical solution. To illustrate this, we consider the semi-infinite metal located in $x<0$ with $r_{s}=4$, and choose $\lambda_{w}=0.5$ for the QHT. The system is driven by a uniform electric field in the $x$ direction at $\omega=0.8 \omega_{p}$. The calculated $n_{1}$ and $n_{1}^{\prime}$ are plotted in Fig. 9. With the simple BC, two different solutions are demonstrated. Additionally, the oscillations of $n_{1}^{\prime}$ in the tail region, due to the interference between the unphysical mode $k_{\mathrm{Lx}}^{(3)}$ and the physical mode $k_{\mathrm{Lx}}^{(4)}$, are observed. With the modified BC, we only have one solution. The unphysical mode $k_{\mathrm{Lx}}^{(3)}$ is filtered out, which leads to the absence of the oscillations of $n_{1}^{\prime}$ in the electron-tail region.

\section{APPENDIX B: PROOF OF $\omega_{\mathrm{mp}}^{Q}<\omega_{\mathrm{c}}^{Q}$}

To prove $\omega_{\mathrm{mp}}^{Q}<\omega_{c}^{Q}$, we define the polarization field $\mathbf{P}_{1}$ by $\partial \mathbf{P}_{1} / \partial t=\mathbf{J}_{1}$, neglect the damping in Eq. (9), and rewrite Eq. (9) as

$$
\frac{\partial^{2} \mathbf{P}_{1}}{\partial t^{2}}=\epsilon_{0} \omega_{p}^{2} f_{0}^{2} \mathbf{E}_{1}+\beta^{2} f_{0}^{2} \nabla\left[\frac{N^{(4)}}{f_{0}^{2}}-\frac{N^{(2)}}{f_{0}} \nabla^{2} \frac{1}{f_{0}}\right] \nabla \cdot \mathbf{P}_{1},
$$

where

$$
N^{(4)}=f_{0}^{4 / 3}+\frac{C_{0} v_{F}^{2}}{\beta^{2}} f_{0}^{4 / 3}-\frac{C_{1} v_{F}^{2}}{6 \beta^{2}} f_{0}^{2 / 3}-\frac{C_{3} v_{F}^{2}}{3 \beta^{2}} f_{0}^{2}+\frac{N^{(2)} \nabla^{2} f_{0}}{f_{0}} .
$$

We focus on the one-dimensional (1D) case that the metal is located in $x<0$, and all relevant quantities only depend on $x$. In the absence of the external charges, the displacement field $\mathbf{D}_{1}=\epsilon_{0} \mathbf{E}_{1}+\mathbf{P}_{1}$ is constant throughout the $1 \mathrm{D}$ space due to Gauss's law. At the MP resonance, a dipolelike charge distribution is established in the surface region, which indicates that $\mathbf{E}_{1} \rightarrow 0$ and $\mathbf{P}_{1} \rightarrow 0$ as $x \rightarrow \pm \infty$. Thus, there is $\mathbf{P}_{1}=-\epsilon_{0} \mathbf{E}_{1}$. These considerations and Eq. (B1) directly lead to that $\mathbf{P}_{1}$ at the MP resonance should satisfy

$$
\frac{1}{f_{0}^{2}} \frac{\partial^{2} \mathbf{P}_{1}}{\partial t^{2}}=-\omega_{p}^{2} \mathbf{P}_{1}+\beta^{2} \frac{\partial}{\partial x}\left\{\left[\frac{N^{(3)}}{f_{0}^{2}}-\frac{N^{(2)}}{f_{0}} \frac{\partial^{2}}{\partial x^{2}} \frac{1}{f_{0}}\right] \frac{\partial \mathbf{P}_{1}}{\partial x}\right\} .
$$

Then, evaluating $\int d x\left[\frac{\partial \mathbf{P}_{1}^{*}}{\partial t} \times\right.$ Eq. (B2) $]+$ H.c., we arrive at

$$
I(\infty)-I(-\infty)=\frac{d U}{d t}
$$

where

$$
\begin{aligned}
I= & {\left[\frac{N^{(3)}}{f_{0}^{2}} \frac{\partial \mathbf{P}_{1}^{*}}{\partial t} \frac{\partial \mathbf{P}_{1}}{\partial x}-\frac{N^{(2)}}{f_{0}} \frac{\partial \mathbf{P}_{1}^{*}}{\partial t}\left(\frac{\partial^{2}}{\partial x^{2}} \frac{1}{f_{0}} \frac{\partial \mathbf{P}_{1}}{\partial x}\right)\right.} \\
& \left.+N^{(2)}\left(\frac{\partial}{\partial x} \frac{1}{f_{0}} \frac{\partial \mathbf{P}_{1}^{*}}{\partial t}\right)\left(\frac{\partial}{\partial x} \frac{1}{f_{0}} \frac{\partial \mathbf{P}_{1}}{\partial x}\right)\right]+ \text { H.c., } \\
U= & \int_{-\infty}^{\infty}\left[\frac{1}{\beta^{2} f_{0}^{2}}\left|\frac{\partial \mathbf{P}_{1}}{\partial t}\right|^{2}+\frac{\omega_{\mathbf{P}}^{2}}{\beta^{2}}\left|\mathbf{P}_{1}\right|^{2}\right. \\
& \left.+\frac{N^{(3)}}{f_{0}^{2}}\left|\frac{\partial \mathbf{P}_{1}}{\partial x}\right|^{2}+N^{(2)}\left|\frac{\partial}{\partial x}\left(\frac{1}{f_{0}} \frac{\partial \mathbf{P}_{1}}{\partial x}\right)\right|^{2}\right] d x .
\end{aligned}
$$

Due to the harmonic time dependence of $e^{-i \omega_{\mathrm{mp}}^{Q}}$, we have $d U / d t=0$ and, accordingly,

$$
I(+\infty)-I(-\infty)=0 .
$$

Equation (B5) serves as the necessary condition for the existence of the MP resonance, which leads to $\omega_{\mathrm{mp}}^{Q}<$ $\operatorname{Min}\left(\omega_{c}^{Q}, \omega_{p}\right)$, as explained in the following. We first focus on the bulk region with $x \rightarrow-\infty$. Clearly, if $\omega_{\mathrm{mp}}^{Q}<\omega_{p}$, $I(-\infty)=0$ since the longitudinal modes all exponentially decay. If $\omega_{\mathrm{mp}}^{Q}>\omega_{p}$, the existence of the bulk plasmon mode leading to $\mathbf{P}_{1} \propto e^{-i \alpha_{1} x}$, where $\alpha_{1}$ is a positive real number representing the plasmon mode propagating towards the $-x$ direction. It is then deduced that $I(-\infty) \propto N^{(3)} \alpha_{1}+$ $2 N^{(2)} \alpha_{1}^{3}>0$. Then, considering the electron-tail region with $x \rightarrow+\infty$. If $\omega_{\mathrm{mp}}^{Q}<\omega_{c}^{Q}$, we know that the induced electron density decays faster than $\sqrt{n_{0}}$ according to the knowledge in Sec. VII A, and simply have $I(+\infty)=0$. However, if $\omega_{\mathrm{mp}}^{Q}>\omega_{c}^{Q}$, there is $\mathbf{P}_{1} \propto e^{-k_{Q} x+i \alpha_{2} x}$, where $\alpha_{2}$ is the positive real number due to the mode $k_{\mathrm{Lx}}^{(4)}$. This apparently leads to $I(+\infty) \propto-N^{(2)} \alpha_{2} k_{d}^{2}-2 N^{(2)} \alpha_{2}^{3}<0$. Thus, based on the above discussions, it is clear that Eq. (B5) can only be satisfied when

$$
\omega_{\mathrm{mp}}^{Q}<\operatorname{Min}\left(\omega_{c}^{Q}, \omega_{P}\right),
$$

which of course implies that

$$
\omega_{\mathrm{mp}}^{Q}<\omega_{c}^{Q} .
$$


[1] S. A. Maier, Plasmonics: Fundamentals and Applications (Springer, New York, 2007).

[2] D. K. Gramotnev and S. I. Bozhevolnyi, Nat. Photonics 4, 83 (2010).

[3] E. Prodan, C. Radloff, N. Halas, and P. Nordlander, Science 302, 419 (2003).

[4] D. P. O’Neal, L. R. Hirsch, N. Halas, J. D. Payne, and J. L. West, Cancer Lett. 209, 171 (2004).

[5] H. Xu, E. J. Bjerneld, M. Käll, and L. Börjesson, Phys. Rev. Lett. 83, 4357 (1999).

[6] J. N. Anker, W. P. Hall, O. Lyandres, N. C. Shah, J. Zhao, and R. P. Van Duyne, Nat. Mater. 7, 442 (2008).

[7] M. A. Noginov, G. Zhu, A. M. Belgrave, V. M. Bakker, V. M. Shalaev, E. E. Narimanov, S. Stout, E. Herz, T. Suteewong, and U. Wiesner, Nature (London) 460, 1110 (2009).

[8] R. F. Oulton, V. J. Sorger, T. Zentgraf, R.-M. Ma, C. Gladden, L. Dai, G. Bartal, and X. Zhang, Nature (London) 461, 629 (2009).

[9] D. E. Chang, A. S. Sørensen, E. A. Demler, and M. D. A. Lukin, Nat. Phys. 3, 807 (2007).

[10] C. Kittel, Quantum Theory of Solids (John Wiley \& Sons, New York, 1987).

[11] B. Henrik and K. Flensberg, Many-body Quantum Theory in Condensed Matter Physics: An Introduction (Oxford University Press, Oxford, 2004).

[12] F. J. García de Abajo, Acs Photonics 1, 135 (2014).

[13] X. Zhu, W. Wang, W. Yan, M. B. Larsen, P. Boggild, T. G. Pedersen, S. Xiao, J. Zi, and N. A. Mortensen, Nano Lett. 14, 2907 (2014).

[14] P. Hohenberg and W. Kohn, Phys. Rev. 136, B864 (1964).

[15] W. Kohn and L. J. Sham, Phys. Rev. 140, A1133 (1965).

[16] E. Runge and E. Gross, Phys. Rev. Lett. 52, 997 (1984).

[17] in Time-Dependent Density Functional Theory, edited by M. A. L. Marques, C. A. Ullrich, F. Nogueira, A. Rubio, K. Burke, and E. K. U. Gross (Springer, Berlin, 2006), Lecture Notes in Physics, Vol. 706.

[18] J. Tao, X. Gao, G. Vignale, and I. Tokatly, Phy. Rev. Lett. 103, 086401 (2009).

[19] X. Gao, J. Tao, G. Vignale, and I. Tokatly, Phy. Rev. B 81, 195106 (2010).

[20] O. Nicoletti, M. Wubs, N. A. Mortensen, W. Sigle, P. A. van Aken, and P. A. Midgley, Opt. Express 19, 15371 (2011).

[21] J. Kern, S. Grossmann, N. V. Tarakina, T. Häckel, M. Emmerling, M. Kamp, J.-S. Huang, P. Biagioni, J. C. Prangsma, and B. Hecht, Nano Lett. 12, 5504 (2012).

[22] K. J. Savage, M. M. Hawkeye, R. Esteban, A. G. Borisov, J. Aizpurua, and J. J. Baumberg, Nature (London) 491, 574 (2012).

[23] J. A. Scholl, A. L. Koh, and J. A. Dionne, Nature (London) 483, 421 (2012).

[24] C. Ciracì, R. T. Hill, J. J. Mock, Y. Urzhumov, A. I. FernándezDomínguez, S. A. Maier, J. B. Pendry, A. Chilkoti, and D. R. Smith, Science 337, 1072 (2012).

[25] S. Raza, N. Stenger, S. Kadkhodazadeh, S. V. Fischer, N. Kostesha, A.-P. Jauho, A. Burrows, M. Wubs, and N. A. Mortensen, Nanophotonics 2, 131 (2013).

[26] J. Scholl, A. Garcia-Etxarri, A. L. Koh, and J. A. Dionne, Nano Lett. 13, 564 (2013).

[27] D. Marinica, A. Kazansky, P. Nordlander, J. Aizpurua, and A. Borisov, Nano Lett. 12, 1333 (2012).
[28] T. V. Teperik, P. Nordlander, J. Aizpurua, and A. G. Borisov, Phys. Rev. Lett. 110, 263901 (2013).

[29] L. Stella, P. Zhang, F. García-Vidal, A. Rubio, and P. GarcíaGonzález, J. Phys. Chem. C 117, 8941 (2013).

[30] E. Madelung, Z. Phys. 40, 322 (1927).

[31] F. Bloch, Z. Phys. 81, 363 (1933).

[32] S. Raza, G. Toscano, A.-P. Jauho, M. Wubs, and N. A. Mortensen, Phys. Rev. B 84, 121412(R) (2011).

[33] N. A. Mortensen, S. Raza, M. Wubs, T. Søndergaard, and S. I. Bozhevolnyi, Nat. Commun. 5, 3809 (2014).

[34] A. D. Boardman, Electromagnetic Surface Modes (John Wiley and Sons, Chichester, 1982).

[35] A. D. Boardman and B. V. Paranjape, J. Phys. F: Met. Phys. 7, 1935 (1977).

[36] A. Eguiluz and J. J. Quinn, Phys. Rev. B 14, 1347 (1976).

[37] S. C. Ying, Nuovo Cimento B 23, 270 (1974).

[38] G. Toscano, S. Raza, A.-P. Jauho, M. Wubs, and N. A. Mortensen, Opt. Express 20, 4176 (2012).

[39] G. Toscano, S. Raza, S. Xiao, M. Wubs, A.-P. Jauho, S. I. Bozhevolnyi, and N. A. Mortensen, Opt. Lett. 37, 2538 (2012).

[40] Y. Luo, A. I. Fernández-Domínguez, S. A. Wiener, S. A. Maier, and J. Pendry, Phy. Rev. Lett. 111, 093901 (2013).

[41] G. Toscano, S. Raza, W. Yan, C. Jeppesen, S. Xiao, M. Wubs, A.-P. Jauho, S. I. Bozhevolnyi, and N. A. Mortensen, Nanophotonics 2, 161 (2013).

[42] F. J. García de Abajo and A. Howie, Phys. Rev. Lett. 80, 5180 (1998).

[43] B. Gallinet, A. M. Kern, and O. J. F. Martin, J. Opt. Soc. Am. A 27, 2261 (2010).

[44] J. Jung and T. Søndergaard, Phys. Rev. B 77, 245310 (2008).

[45] W. Yan, N. A. Mortensen, and M. Wubs, Phys. Rev. B 88, 155414 (2013).

[46] R. Ruppin, Phys. Rev. Lett. 31, 1434 (1973).

[47] F. J. García de Abajo, J. Phys. Chem. C 112, 17983 (2008).

[48] C. Ciracì, E. Poutrina, M. Scalora, and D. R. Smith, Phys. Rev. B 85, 201403(R) (2012).

[49] A. I. Fernández-Domínguez, A. Wiener, F. J. García-Vidal, S. A. Maier, and J. B. Pendry, Phys. Rev. Lett. 108, 106802 (2012).

[50] T. Christensen, W. Yan, S. Raza, A. P. Jauho, N. A. Mortensen, and M. Wubs, Acs Nano 8, 1745 (2014).

[51] R. Esteban, A. Borisov, P. Nordlander, and J. Aizpurua, Nat. Commun. 3, 825 (2012).

[52] C. Schwartz and W. Schaich, Phys. Rev. B 26, 7008 (1982).

[53] C. David and F. J. García de Abajo, Acs Nano 8, 9558 (2014).

[54] E. Zaremba and H. Tso, Phy. Rev. B 49, 8147 (1994).

[55] N. Lang and W. Kohn, Phys. Rev. B 1, 4555 (1970).

[56] C. von Weizsäcker, Z. Phys. 96, 431 (1935).

[57] Y. Tomishima and K. Yonei, J. Phys. Soc. Jpn. 21, 142 (1966).

[58] J. R. Smith, Phys. Rev. 181, 522 (1969).

[59] G. Manfredi and F. Haas, Phys. Rev. B 64, 075316 (2001).

[60] S. A. Khan and M. Bonitz, Complex Plasmas, edited by M. Bonitz, J. Lopez, K. Becker, and H. Thomsen (Springer, New York, 2013), pp. 103-152.

[61] S. Conti and G. Vignale, Phys. Rev. B 60, 7966 (1996).

[62] I. Tokatly and O. Pankratov, Phys. Rev. B 60, 15550 (1999).

[63] I. Tokatly and O. Pankratov, Phys. Rev. B 62, 2759 (2000).

[64] J. F. Dobson and H. M. Le, Phys. Rev. B 66, 075301 (2002). 
[65] I. V. Tokatly, Phys. Rev. B 71, 165105 (2002).

[66] I. V. Tokatly, Phys. Rev. B 75, 125105 (2007).

[67] C. A. Utreras-Diaz, Phys. Rev. B 36, 1785 (1986).

[68] A. Chizmeshya and E. Zaremba, Phys. Rev. B 37, 2805 (1987).

[69] G. Toscano, C. Rockstuhl, F. Evers, H. Xu, N. A. Mortensen, and M. Wubs, arXiv:1408.5862.

[70] P. Halevi, Phys. Rev. B 51, 7497 (1995).

[71] L. H. Thomas, Proc. Cambridge Philos. Soc. 23, 542 (1926).

[72] E. Fermi, Acad. Naz. Lincei 6, 602 (1927).

[73] E. P. Wigner, Phys. Rev. 46, 1002 (1934).

[74] N. D. Mermin, Phys. Rev. B 1, 2362 (1970).
[75] J. Jin, The Finite Element Method in Electromagnetics (Wiley, New York, 2002).

[76] C. M. Bender and S. A. Orszag, Advanced Mathmatical Methods For Scientists and Enginers (McGraw Hill, Singapore, 1984).

[77] H. Ehrenreich and M. H. Cohen, Phys. Rev. 115, 786 (1959).

[78] A. Liebsch, Phys. Rev. B 36, 7378 (1987).

[79] K. D. Tsuei, E. W. Plummer, A. Liebsch, E. Pehlke, K. Kempa, and P. Bakshi, Surf. Sci. 247, 302 (1991).

[80] A. Liebsch, Phys. Rev. B 48, 11317 (1993).

[81] P. J. Feibelman, Prog. Surf. Sci. 12, 287 (1982).

[82] P. Apell and D. R. Penn, Phys. Rev. Lett. 50, 1316 (1983). 